\title{
Reengineering the Booch Component Library ${ }^{1}$
}

\author{
Jordi Marco, Xavier Franch \\ \{jmarco | franch\}@1si.upc.es \\ Dept. Llenguatges i Sistemes Informàtics (LSI) \\ Universitat Politècnica de Catalunya (UPC) \\ Jordi Girona 1-3 (Campus Nord). E-08034 Barcelona, Catalunya (Spain) \\ FAX: 34-3-4017014. Phone: 34-3-4017006
}

\begin{abstract}
Component-based software construction heavily relies on the ability of reusing components from a library with as little effort as possible. Among others, valuable features for reusing from a component library are: adaptability to many contexts, extensibility, abstraction and high level of robustness with respect to changes in some of their components. In this paper we study one of the most widely used component library for Ada 95, the Grady Booch's one, mainly in relation to these features. The hierarchy of the Booch component library is organised into three main super-classes: Containers, Support and Graphs, which have a common parent-class. Our study focuses on the Container-classes family, which present some drawbacks mainly due to the fact that some parent-classes depend on the concrete form of their children-classes. We state first the problems and we propose next a solution centred on changing the Containers base class. This new version of the Containers class offers a new concept, namely shortcut, that allows not only to avoid the dependencies between parent-classes and their children classes, but also offers some additional advantages, remarkably improving the efficiency of components and increasing the possibilities of reusing from the library in contexts with strong efficiency requirements. Most of the ideas of this work can be generalised to other component libraries.
\end{abstract}

\section{Introduction}

Component-based software development [Jaz95, Sit94] heavily relies on the ability of reusing components from a library with as little effort as possible. Reusing from a library can only take place when the library fulfils some nice properties. To name a few:

- It should be versatile, offering a wide range of components with different functionalities, and also with the same functionality but different implementation strategies (each one suited for particular efficiency requirements).

- It should be open, in the sense that its users should be able to add easily other components to adapt the library to their own context.

- It should be stable, to avoid new future releases forcing changes on existing software.

- It should be abstract enough, to allow easy use.

- And of course, it should be correct and properly documented.

One of the best well-known component libraries for Ada 95 [Ada95] is the Grady Booch's one. This library was originally created for Ada 83 [Boo87] and reengineered first for C++ [BV90]

\footnotetext{
${ }^{1}$ This work has been partially supported by the Spanish research programme CICYT under contract TIC97-1158.
} 
and later on for Ada 95 [BWW99]. This library fulfills many of the properties listed above, and it has proven to be very useful in the development of component-based software. However, it also presents some drawbacks that make it not as powerful as it could be with respect to the following criteria:

- Versatility. It offers a wide range of components, but it is not as versatile as one could expect, because all its components have just a single implementation.

- Efficiency. Not only does the lack of many implementations provoke a loss of efficiency in some contexts, but some of these implementations are not efficient enough, because component interfaces do not allow direct access to certain parts of the implementation.

- Openess. New components and implementations for components could be added, but implementations for already existing components should look very similar to the existing ones, due to the internal structure of the library.

- Stability. Some feasible changes on implementations can damage existing programs, because component definitions make use in some places of features that are inherent of proper implementations.

- Usage. The mixture of specification and implementation characteristics in component definitions interferes with the easy understanding and use of the components therein.

The purpose of this paper is to make reengineering on the Booch library to solve these problems. The proposal is based mainly on changing a particular base class, Containers, in such a way that all the dependencies on a concrete implementation disappear. This new class allows easy integration in the library of new components and also of new arbitrary implementations for every component, even the already existing ones. Moreover, the new Containers class offers an enlargement in its interface that make its derived classes more efficient in a wider range of contexts. All the changes in the library do not affect existing programs, which are using it; it only needs a recompilation step. However, existing programs could be improved with this new version of Containers to be more efficient.

The rest of the paper is organised as follows. First, in Sect. 2, we analyse the Booch component library for Ada 95 describing the advantages and drawbacks that it presents. Then, in Sect. 3, we introduce the main features of a new Containers class that allows solving the problems that the Booch library presents. Section 4 explains the implementation details of the new Containers class. Next, in Sect. 5, we show the necessary modifications of the Containers children-classes and an example. Finally, in Sect. 6, we give the conclusions and future work.

\section{Analysing the Booch component library for ADA 95}

The Booch component library is one of the best-known component libraries for Ada 95 [Ada95]. This library was originally created for Ada 83 [Boo87] and reengineered first for C++ [BV90] and later on for Ada 95 [BWW99]. The Ada 95 version of the Booch components is organised into three main super-classes: Containers, Support and Graphs, which have a common parent-class $B C$. The base class $B C$ has no functionality at all, it only provides the definition of the common exceptions. The Containers category of classes provides a wide range of structural abstractions (lists, bags, sets, collections, etc.) using many widespread 
implementation techniques (chaining, hashing, search trees and so on). Figure 1 shows the main hierarchy of these components; their code is available at [BWW99].

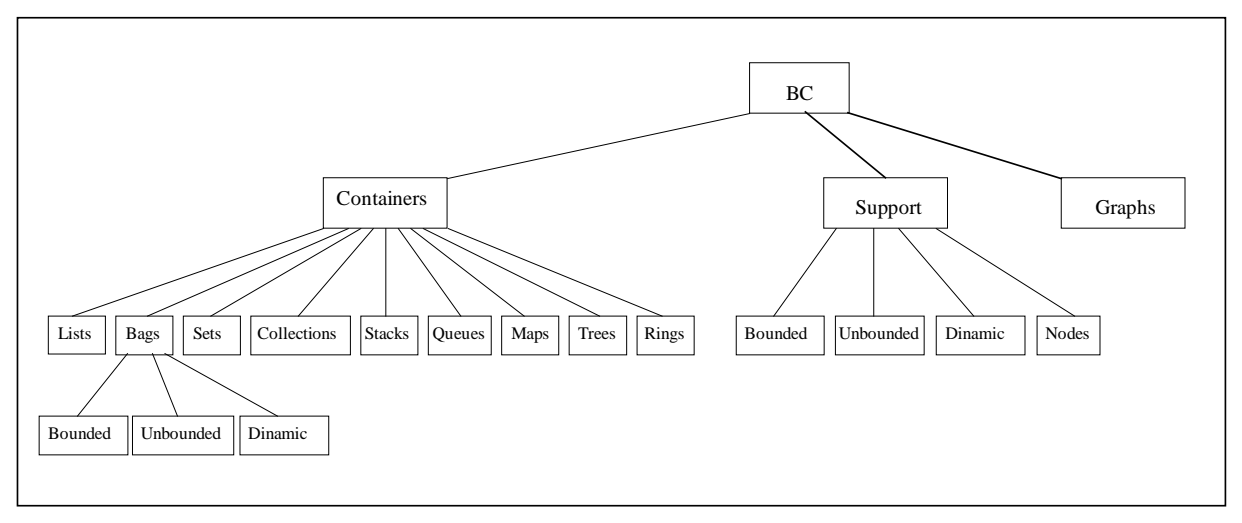

Figure 1: Hierarchy of the Booch component classes (excerpt).

Our study is centred on the Container family of classes, which presents some drawbacks that decrease the potential of reusability of this component library. Most of the problems arise because some parent-classes depend on the concrete implementation of their children-classes. To make it clearer, Fig. 2 shows a typical situation in this library, in which the BC.Containers.Bags class depends on the concrete form of its children classes Bounded, Unbounded and Dynamic, which are hashing tables. Notice that the type definition of Bag_Iterator ${ }^{2}$ forces all the Bags children to be implemented by means of a hashing table. This restriction interferes with the possibility of extending the class hierarchy or changing the concrete form of one of its children. All these dependencies exist because iterators are strongly dependent on the concrete container implementation. Similar dependencies can be also found between the classes Maps, Sets, Queues, etc., and their respective children. Therefore, to solve the problems we clearly need to make iterators independent of the specific container.

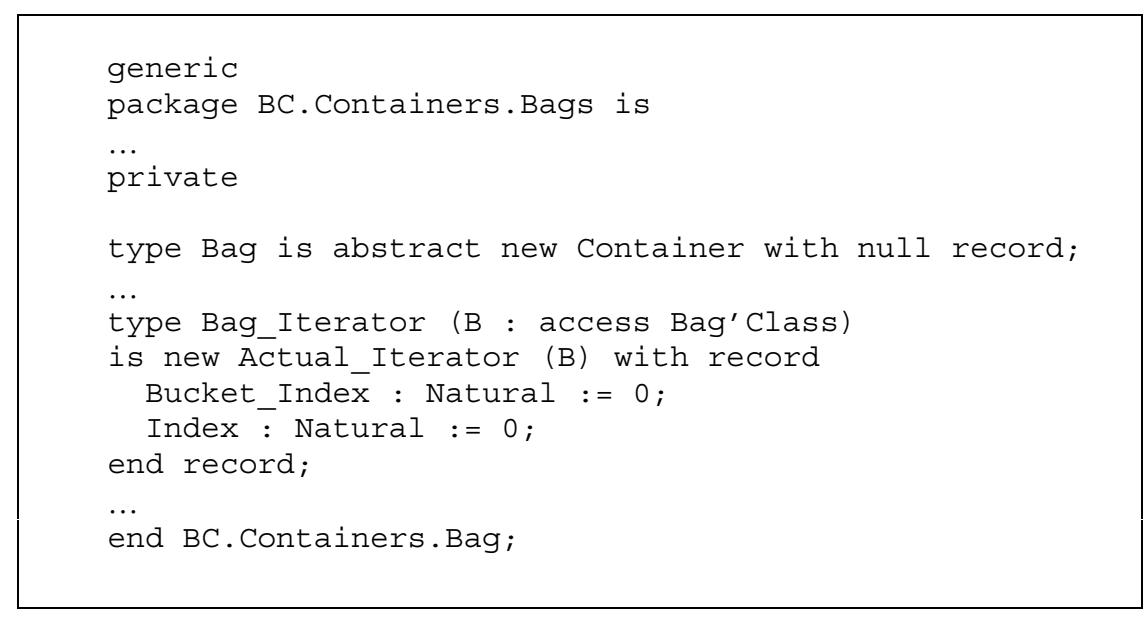

Figure 2: Extract of the generic package BC.Containers.Bags.

\footnotetext{
${ }^{2}$ An iterator is a separate abstract data type, found in most of the existing comercial libraries, that allows iterating through all the items in a structure. Its existence and good behaviour is critical for a library being competitive, and this is why all of the most important component libraries offer them in one way or another.
} 
To sum up, the main problems in this library are:

- The hierarchy is not robust enough with respect to changes in some of their components: changes in a component require the modification of other components. This is due to the implementation dependency mentioned above. For instance, changing the current hashing implementation of the BC.Containers.Bags.Bounded by an implementation (bounded) with a binary search tree (for instance, because elements must be obtained in some order), the implementation of the type Bag_Iterator, defined in the BC.Containers.Bags class, must be changed, and the implementation of its operations as well.

- Moreover, this hierarchy restricts to a single set of possible implementations for some of the different structure abstractions. This is a serious drawback because some of the implementations provided therein can be inefficient in some contexts (we have already mentioned ordered traversal of Bags). For instance, it is not possible to have different implementations of the class BC.Containers.Bags.Bounded, because it is not implementation-independent, and hence it forces a concrete implementation strategy (hashing). This problem could be solved adding another level in the hierarchy, making the class abstract and defining their concrete children, but it is not possible without changing other parts of the hierarchy, because of the implementation dependency again.

- Low level of abstraction makes the usage of the implementation harder. This happens when dealing with iterators. The iterator type and its operations are strongly dependent on the concrete implementation of the underlying structure. As a consequence, for every concrete implementation of a children-class a new Actual_Iterator type must be defined and its operations must be overridden. This approach, which is different from many other libraries, prevents the easy usage of the iterator facility.

- It is not only the lack of multiple implementations for components that damage efficiency, but also some of the iterator operations have lineal cost in the worst case with respect to a certain parameter (although their amortised cost is constant). For instance, as shown in Fig. 3, the Reset operation could have linear cost with respect to the Number_of_Buckets. A similar problem occurs in the Next operation.

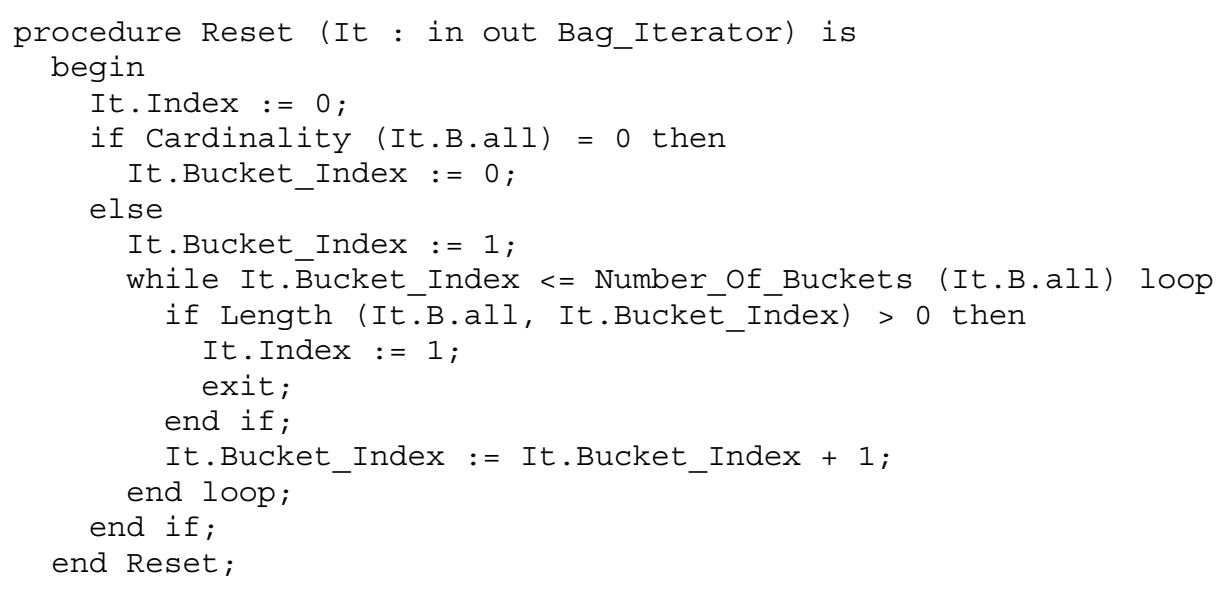

Figure 3: Reset operation's code of the generic package BC.Containers.Bags. 
- In many contexts in which components often encapsulate data structures, reusability can be damaged due to efficiency requirements: even if a component fulfils a required functionality, the time complexity of its operations may be inadequate given the context in which it should be integrated (either considering them individually or when combining them to build more complex components). The access by means of the operations offered by a component may be costly if the logical layout of the data structure is used; if fast access is required, it becomes necessary to look up the item using directly a reference to it.

It can be argued that these disadvantages are strong enough to rebound the use of the Booch library. However, this is not the case; this library offers several advantages that make it very useful, mainly:

- A large amount of robust and well-designed components with appropriate algorithms and data structures.

- It is a well-known library supported by documentation and books.

- It is freeware.

- It has several and complete testing packages for its components.

Given the above advantages we think it is worth improving this library by solving the above mentioned problems, instead of discarding it and looking for building a brand new one.

\section{A new Containers class}

The goal of this paper is to improve the containers-class family of Booch components library to avoid the problems shown in the previous section. Our approach consists on changing the base class Containers in such a way that the items will be stored in a generic container. Then iterators are implemented in this generic container and so they are independent of the concrete implementation of the children-classes. This generic container will offer efficient access paths to the items therein, which we call shortcuts. The Containers children-classes must store the shortcuts (that allow access to items) instead of the items themselves. In addition, the users can obtain an alternative, efficient and abstract way to access to the items stored in the structure.

The children classes of the new Containers class inherit from it the shortcuts and the operations. Therefore, the items stored therein can be accessed not only by using the operations given in the former specification, but also by means of other new ones that use the efficient paths. This results very useful whenever the operations are considered too expensive; on the other hand, since the addition of shortcuts does not affect the former behaviour of the existing operations, they can be used in the other cases as well.

Shortcuts solve the problems mentioned in the previous section:

- The items in the container can be accessed without knowing how they are stored in the container and, therefore, without knowing the underlying representation of the container (with arrays, pointers, linked, in tree-form, ...). Therefore, many implementations can exist together for the same type of container. 
- The access to the items in a container by means of a shortcut is achieved in constant time $\mathrm{O}(1)$, making it possible to reuse containers even with high efficiency constraints.

- The addition of shortcuts to a container does not modify its functional behaviour. This is assured by incorporating the concept of shortcut into the formal specification of the container (see [FM00] for more details). Preservation of behaviour makes possible the substitution of old components by new ones.

Shortcuts are created and destroyed dynamically as items are inserted to and removed from a container; every item is bound to one (and only one) item. The shortcut bound to an item remains the same while it is inside the container, even if the underlying representation requires rearrangements. The access to the items by means of shortcuts is safe because meaningless access to them is avoided: our approach avoids dangling shortcuts or out-of-date ones.

\section{Implementing the new Containers class with shortcuts}

The essential point consists in implementing the generic container with a mapping from shortcuts to items. At the same time, items in the children components must be substituted by the shortcut that identifies them. The mapping from shortcuts may be implemented both using dynamic storage or an array; shortcuts are implemented then as pointers or cursors (i.e., array positions), respectively. Fields to obtain (in constant time) the shortcuts to the last item stored and to the first and the last item in the iterator order are necessary too; the field first to reset an iterator and the field last to add an item after the previous last item in the iterator order (which is not the same that the last item stored because it can have been added anywhere).

Released shortcuts must be available somehow to allow further reassignment, provided that there are not extra copies of it. When an item is removed from the container, it is marked as deleted. The corresponding shortcut can only be reused when there are no references to it. Therefore, memory management should be incorporated in the implementation. In this paper shortcuts are implemented by pointers reusing a particular memory manager offered by the Booch's library itself.

To assure efficient and independent iterators we need to create a double-linked list of shortcuts, which means that we need $2 * N *$ space(pointer) $+N * \operatorname{space}$ (shortcut) extra space (where $N$ is the number of items in the container). Then, the iterators are implemented as shortcuts. The waste of this extra space offers a lot of benefits: the iterators are independent of the concrete form of the container, the efficiency of all the iterator operations is constant even in the worst case, and even this waste of space will generate a later saving, when shortcuts substitute identifiers (generally strings, which require more space than pointers or integers) in outer references from programs or other data structures.

Let's now establish formally the equation that assures the saving of space. Let $N$ be the total number of items in the container and $R$ the total number of external references. Since generally,

$$
\operatorname{space}(\text { item }) \geq \operatorname{space}(\text { pointer) }
$$

then $\exists k \geq 1$ s.t. $\operatorname{space}($ item) $\geq k *$ space(pointer) 
and since space(shortcut) $=2 * \operatorname{space}$ (pointer) (see below) space is really saved when the relationship

$$
R * \operatorname{space}(\text { item }) \geq 4 * N * \operatorname{space}(\text { pointer })
$$

Holds, which is satisfied when the following relationship holds:

$$
R * k \geq 4 * N \text {. }
$$

Figure 4 shows the implementation of the types of the new class BC.Containers. The Shortcut type is implemented with a record with two fields: the first one is an access to the Container to which the shortcut is associated and the last one is a pointer (reused from BC.Smart) to the node where the item is stored. The Node type is a record with four fields: the first one is the item itself, the second and the third ones are pointers to maintain doubled-linked list of items and the last one is a boolean to mark the item as deleted when it has been logically removed but there are still some shortcuts that refer to it. The Container type is a record that maintains the corresponding fields for the last, first and last added items, and an additional field to obtain the number of items therein in constant time.

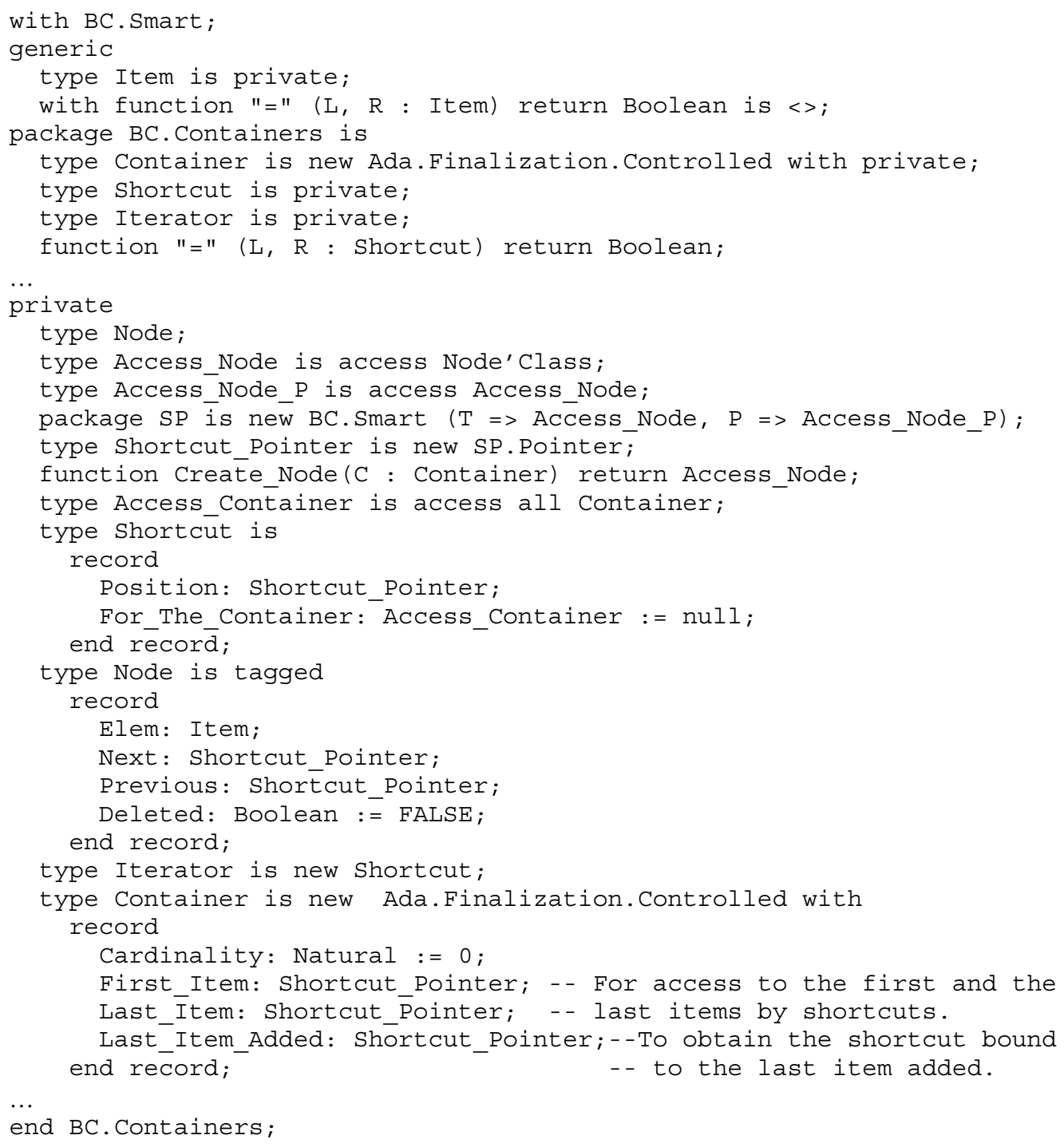

Figure 4: New BC.Containers class. 
This scheme also works, without further considerations, in the case of the linear containers, such as stacks, lists, and so on. Figure 5, on the left, shows this situation with stacks. Now, the stack contains just shortcuts, and the objects are stored in a generic container, directly accessible by means of the shortcut. In the case of types accessed by some kind of key, like bags, maps, etc., we need to define the functions required for the concrete implementation over the shortcuts (for instance, the equality function and, in case of a hashing table, the hash function). These new functions will consist in applying the original function to the item associated to the shortcut. The scheme is shown in figure 5, on the right, and the Ada 95 code can be found in the appendix.
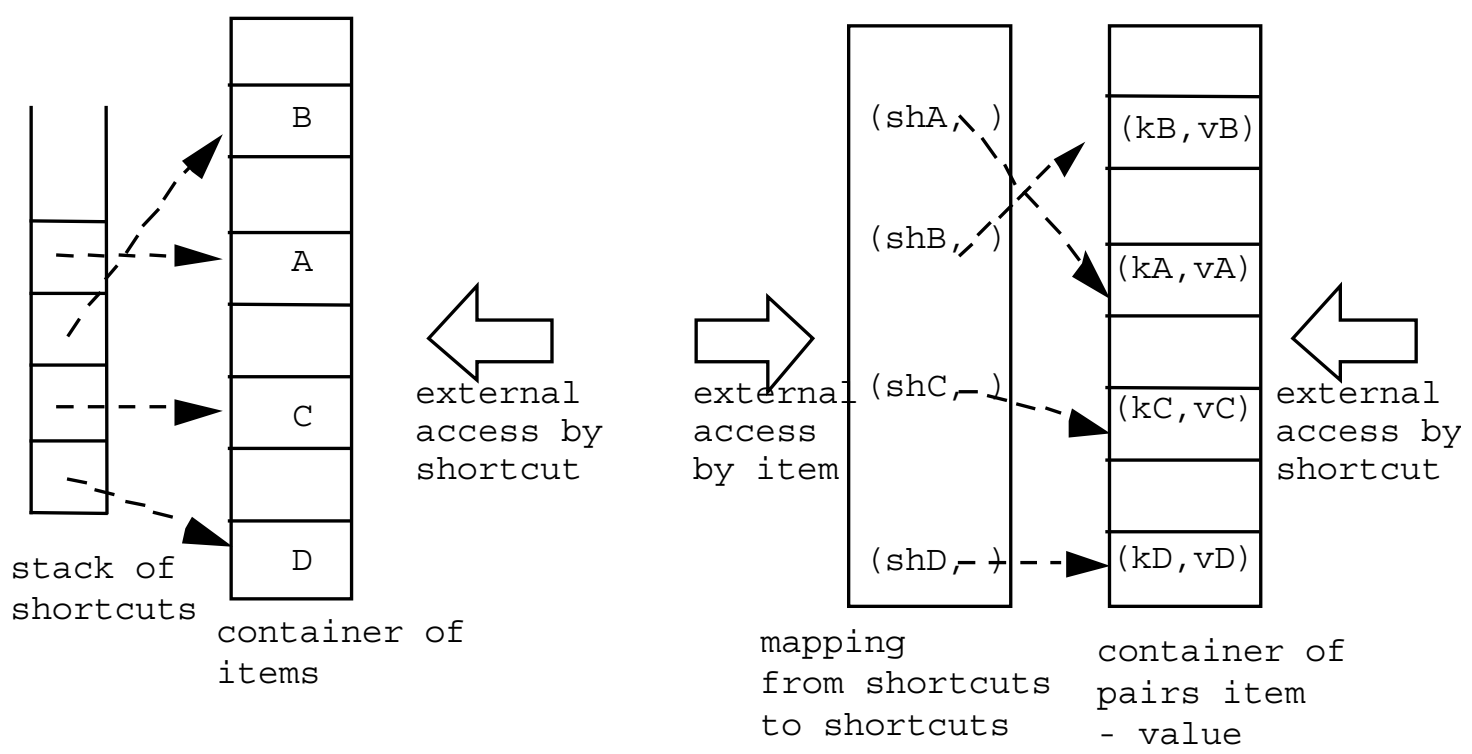

Figure 5: Adding shortcuts to linear structures (left) and to mappings (right).

The new Containers class includes, in addition to the operations offered by the old one, seven new ones shown below. The first three ones are public while the others are private and, as a consequence, can only be used by the children classes (Fig. 6 shows the signature of these operations; see the appendix for a complete implementation). Their behaviour is as follows:

- $A d d$ adds the given item into the container in the last position (with respect to the iterator order). To be used in concrete containers implementations that do not require a special traversing order.

- Add_After adds the given item after the one bound to the given shortcut (with respect to the iterator order). If the shortcut is undefined, an exception arises. To use in concrete containers implementations that require a special traversing order.

- Shortcut_To_The_Last_Item_Added returns the shortcut that allows accessing to the last item added (using Add or Add_After) into the container. If there are no items in the container, it returns an undefined shortcut. This operation is required to obtain the shortcuts from the data structure; once obtained, it can be stored in other data structures and then coupling of structures without duplication of items is possible.

- Item_Of returns the item bound to the given shortcut. If the shortcut is undefined, an exception arises.

- Defined returns true if there is an item associated to the given shortcut, otherwise returns false. 
- Remove removes from the container the item associated to the given shortcut. If the shortcut is undefined, an exception arises.

- Node_Of returns an access to the node where the item associated to the given shortcut is stored. If the shortcut is undefined, an exception arises. This operation is required in order to access to the values that have been stored together with the item, if it is the case. In the children classes, public efficient operations to access to these values by shortcuts can be defined. To allow this, the Node type is tagged and the children classes can define a new Node type with the necessary extra fields; in this case, the Create_Node function (see Fig. 4) has to be overridden.

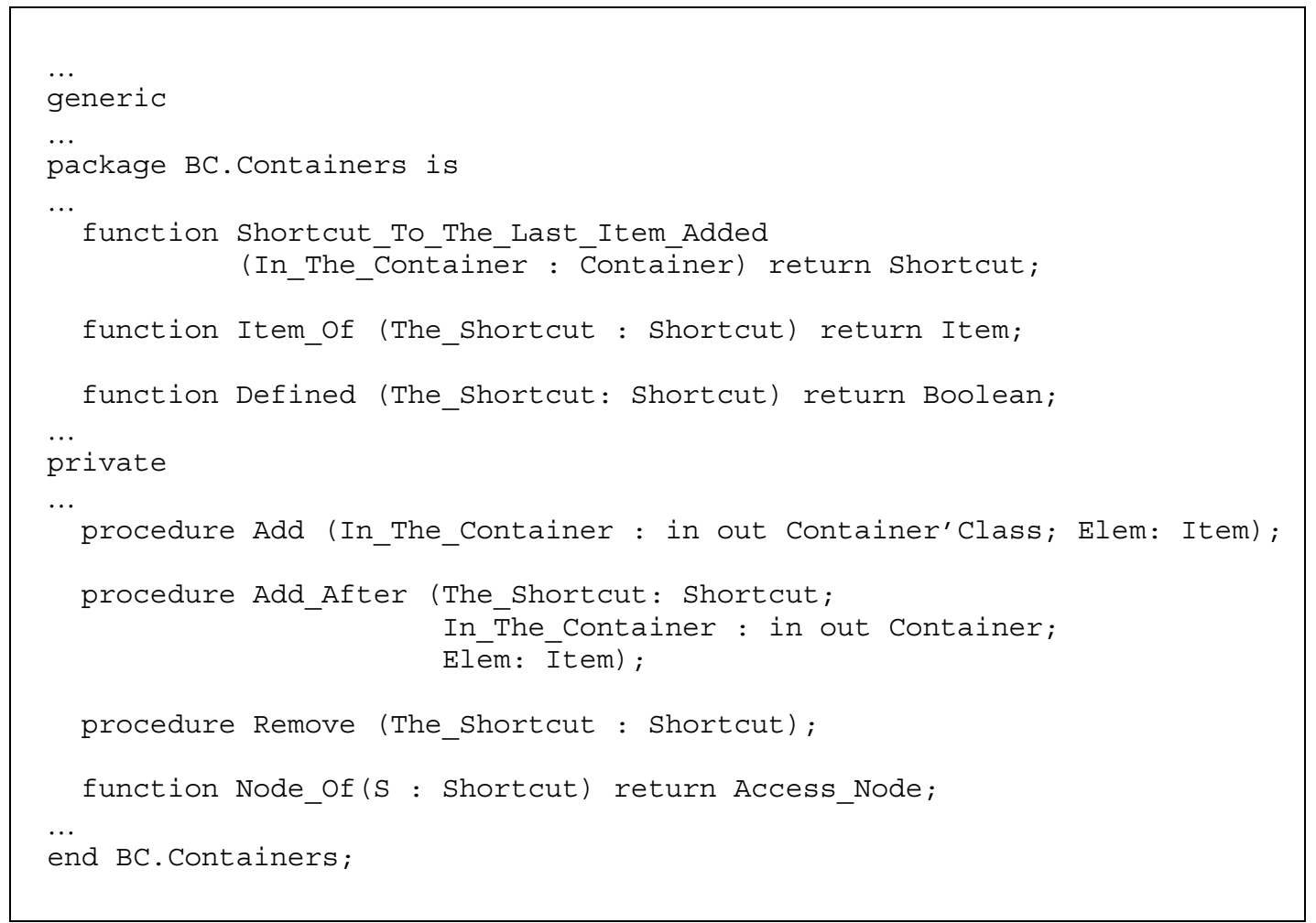

Figure 6: Signature of the new operations of the BC.Containers class.

We remark that the cost of all these operations as well as all the iterator operations is constant even in the worst case. Another important feature is that the operations Shortcut_To_The Last_Item_Added, Item_Of and Defined are public and they are inherited by the children-classes of Containers. In consequence, all the children classes offer shortcuts and their users can take profit of them to improve the efficiency of their programs.

The complete implementation can be found not only in the appendix but also in ftp://ftp.lsi.upc.es/pub/users/jmarco/.

\section{Using the new Containers class}

In this section, we explain the steps required to modify the Containers children-classes to adapt them to the new Containers class. First, we explain the general changes required and then we applied the required changes to a concrete example, the class BC.Containers.Bags.Bounded and its ancestors. 
In the general case, the changes required in the Containers children-classes are:

- Remove the specification and the implementation of the concrete iterators (i.e., the new Actual_Iterator).

- Modify the operations that take into account features that are tight to the concrete implementation of the new Actual_Iterator.

- In the class where the container is implemented we must:

- Replace the items by shortcuts.

- If there are elements to store together with the item (as in the example below), then define a new Node type and override the function Create_Node to initialise these elements.

- If the existing implementation needs some specific functions (e.g., a hash function in the case of a container implemented by hashing), then redefine them working on shortcuts instead of items.

- For every operation that works with items, add the indirection required to work with shortcuts instead of items.

We present below an example of the (few) changes to be done in the Containers childrenclasses. More precisely, we explain the required changes in the class BC.Containers.Bags and BC.Containers.Bags.Bounded, respectively. Similar changes should be done to the rest of children-classes.

The only required change in the package specification BC.Containers.Bags is to remove the iterators therein, i.e. the new Actual_Iterator type as well as its corresponding operations (Fig. 7 shows the part of the specification that must be removed).

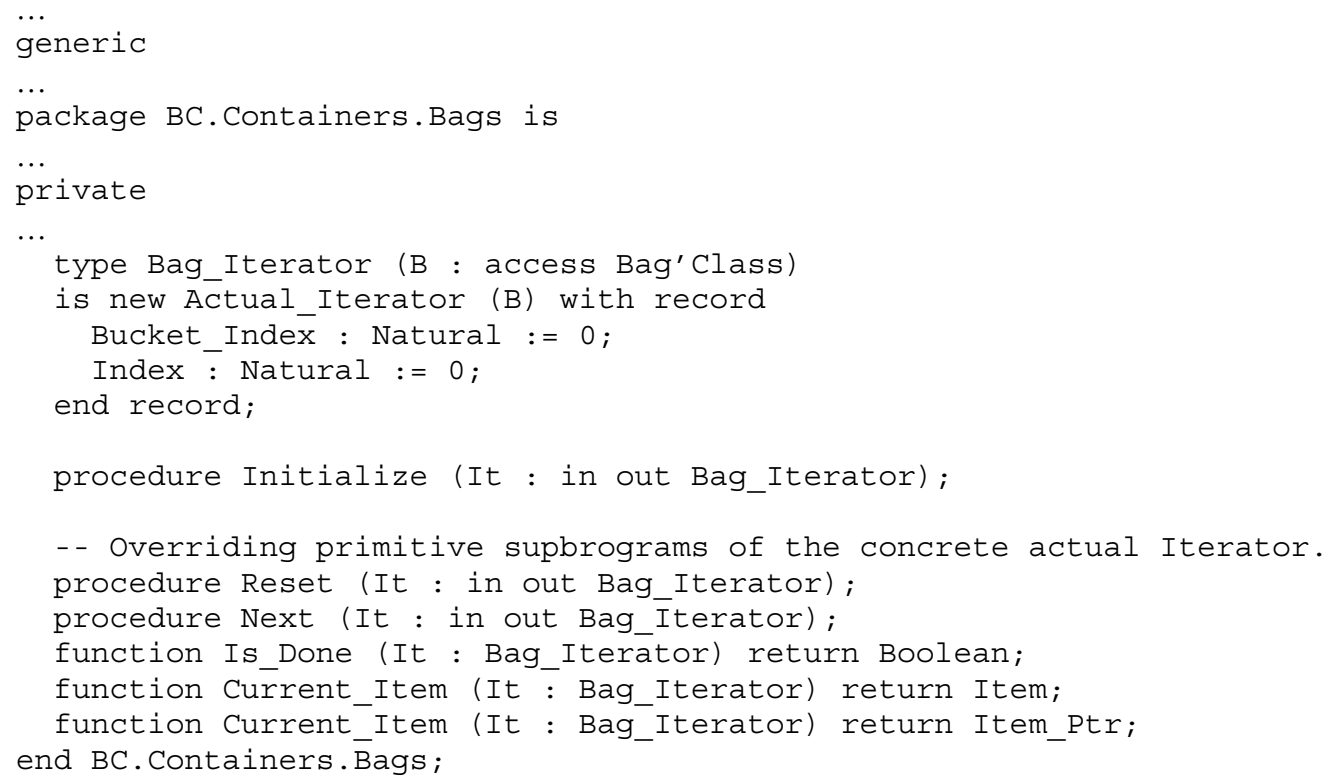

Figure 7: Types and operations of the BC.Containers.Bags class that must be removed. 
The changes required in the package body are: removing the body of all the iterator operations and modifying the Intersection operation, because this operation takes into account that the elements in the hashing table can change their position when removals take place. Therefore the iterator to the next element remains the same. Figure 8 presents the new Intersection operation (the changes are marked with a comment IS NEW) .

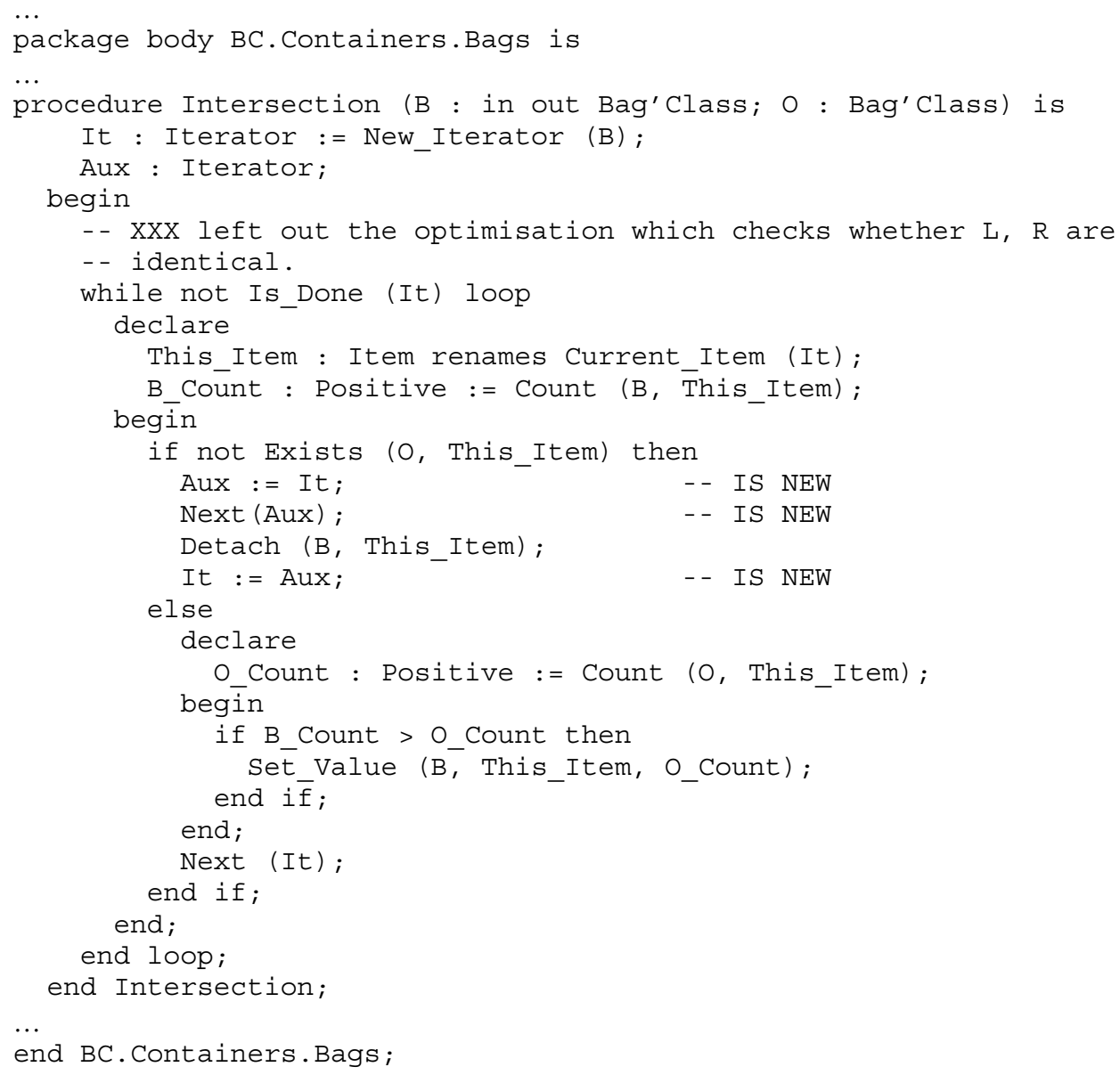

Figure 8: New version of Intersection operation of the BC.Containers.Bag.

The required changes of the Containers.Bags.Bounded are to create the hash table with shortcuts as items and as values (see Fig. 9) and to remove the specification of the functions Item_At and Value_At and of the type Bounded_Bag_Iterator in the specification package. Notice that we need to store the number of copies of an item (because a bag actually owns only one copy of each item and it counts the duplicates). We must then define a new Node type to store the number of occurrences together with the item. In consequence, the function Create_Node (introduced in Sect. 5) must be overridden. We must also define the function "=" and the function New_Hash to take into account the necessary indirection provoked by storing the shortcuts instead of the items. 


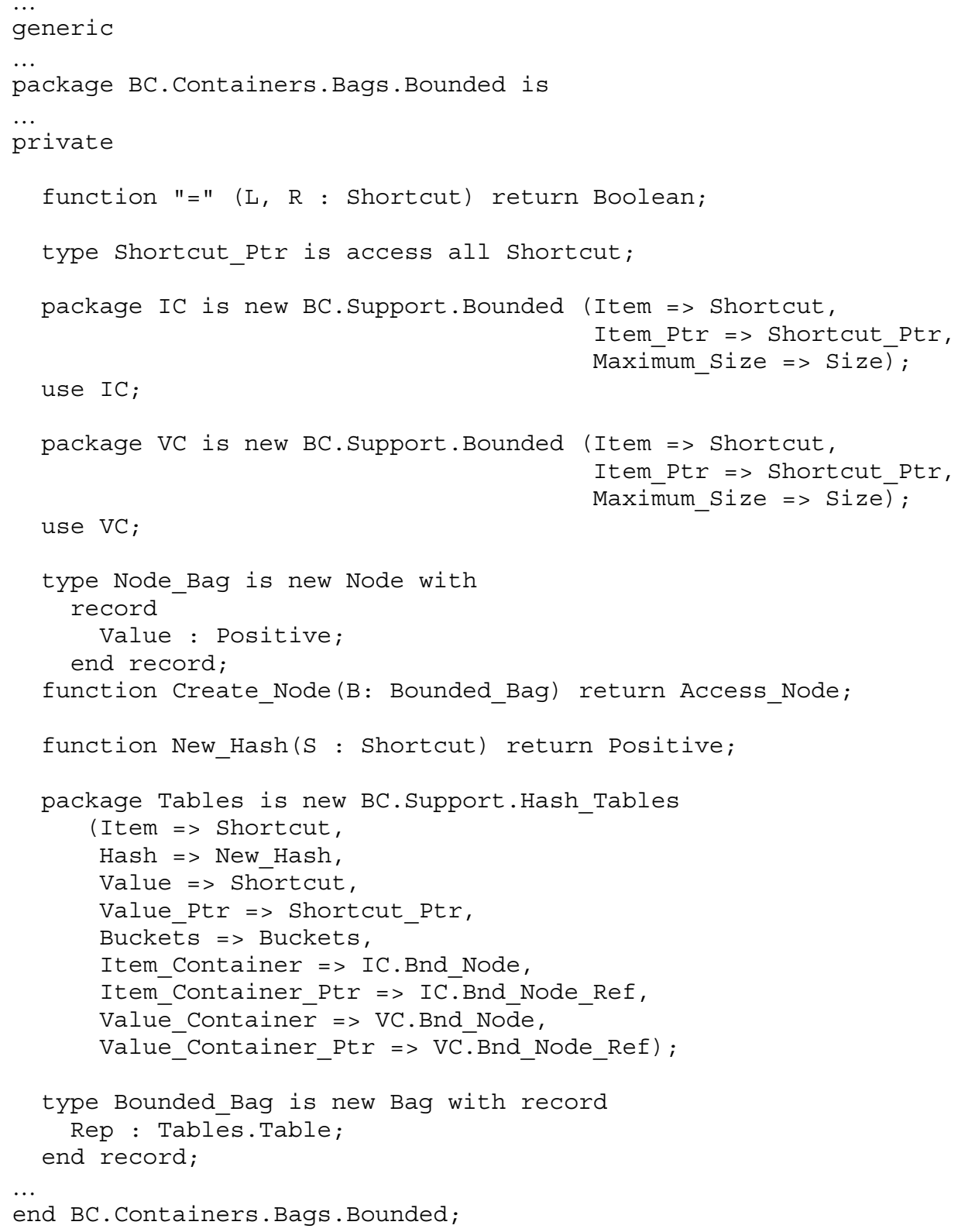

Figure 9: Changes in the package specification BC.Containers.Bags.Bounded.

In the package body, we must add for every operation the corresponding indirection to access to the item besides of the corresponding implementation of Create_Node, "=" and New_Hash. Figure 10 shows the implementation of these three functions and, as an example of how indirections are added, the implementation of the operation $A d d$. In the appendix, we present the whole new Containers class and the classes Containers.Bags and Containers.Bags.Bounded with all the required modifications. 


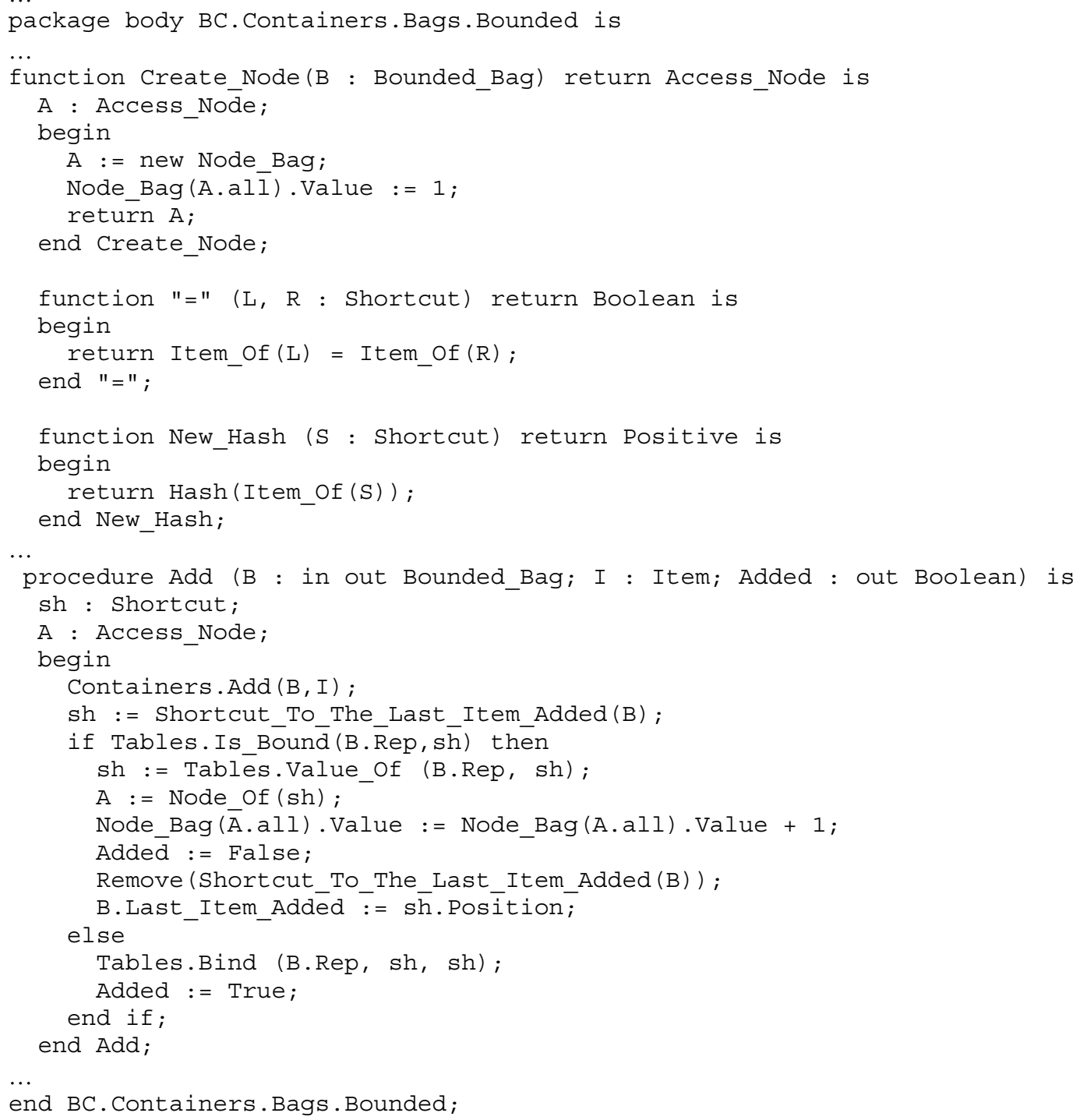

Figure 10: Implementation of the functions Create_Node, "=", New_Hash and of the operation Add of BC.Containers.Bags.Bounded.

In the operation $A d d$, we associate first a shortcut to the item, and then we ask if it is already bound to some item in the hash table. If it is already bound, we must increment the number of occurrences using the previous shortcut associated to the item and remove the temporal one. 


\section{Conclusions}

Component libraries play currently a crucial role on software development. The existence of a wide variety of such libraries, both general-purpose and also field-specific, is becoming essential and its importance will increase in the future. However, nowadays a twofold phenomena occurs. On the one hand, most of the existing libraries present some features that can be improved, concerning quality factors as efficiency, usability, functionality, etc. On the other hand, potential clients of the library tend to simply discard it if it does not fit completely into their context. Our point of view is that both phenomena will be corrected little by little, when maturity in the field will be reached.

Our paper tries to be a contribution to this madurity process. We have presented here a case study of reengineering a good library, the Booch one, that presents a few drawbacks. First, we have enumerated the advantages and disadvantages it presents and then we have proposed a solution to all the drawbacks. The solution is based on designing a new Containers base class that avoids the problems and also offers a new type that implement the concept of shortcut as alternative access path to items in the container. Shortcuts are interesting because, besides of assuring fast access time to items in the container, they are abstract (independent of the implementation of the component), persistent (movements inside the data structure do not affect them), secure (meaningless accesses are not possible) and they preserve behaviour (the new component behaves as the old one). Therefore, both existing and new software can benefit from the nice properties that present the concept of shortcut. We have also shown the few necessary changes in the Containers children-classes to use the new Containers base class. The paper focuses in the technical details, to show the feasibility of the reengineering process and to make explicit the cost of this process.

The library with the modifications presented in this paper can be found in ftp://ftp.lsi.upc.es/pub/users/jmarco/.

We would like to point out the benefits of our approach:

- The Booch library is improved from many points of view: versatility, stability, openess, efficiency and ease of use.

- This improvement has been made in a very comfortable way; with only few changes needed. The core data structures and algorithms are the same without any modification at all.

- The concept of shortcut is general enough to be exported to other libraries. In fact, most of the main existing component libraries offer it in many different ways [MN99, MS96]. The advantage of our proposal is that it provides a systematic way of adding shortcuts to existing libraries, instead of putting them in the library from the very beginning.

- The reengineering process does not interfere with the previous behaviour of the library (both for functionality and for efficiency) and in consequence, existing software that use this library does not need to be modified; only recompilation is needed.

- But existing software could be modified in a methodical way (basically, changing the way of accessing to the structure) to take profit of the new version of the library. New 
software, of course, will be built in general using the new layout of the structure, making intensive use of shortcuts.

- The new library has been tested using the bag_test provided by the original Booch library without any modification.

\section{References}

[Ada95] S. Tucker Taft and R.A. Duff (eds.). Ada 95 Reference Manual. Lecture Notes in Computer Science 1246, Springer-Verlag, 1995.

[Boo87] G. Booch. Software Components with Ada. The Benjamin/Cummings Publishing Company Inc., $2^{\text {nd }}$ edition, 1987.

[BV90] G. Booch and M. Vilot. The Design of the C++ Booch Components. In Proceedings of Conference on Object Oriented-Programming: Systems, Languages and Aplications (OOPSLA), volume 25 of SIGPLAN Notices, pages 1-11. ACM 1990.

[BWW99]G. Booch, D.G. Weller and S. Wright. The Booch Library for Ada 95 (version 1999). Available at http://www.pogner.demon.co.uk/ components/bc/.

[FM00] X. Franch and J. Marco. Adding Alternative Access Paths to Abstract Data Types. To appear in Proceedings of 2000 Information Resources Management Association International Conference (IRMA). To celebrate in May 2000.

[Jaz95] M. Jazayeri. Component Programming - a Fresh Look at Software Components. In Proceedings of $5^{\text {th }}$ European Software Engineering Conference (ESEC), volume 989 of LNCS, pages 457-478. Springer-Verlag, 1995.

[MN99] K. Mehlhorn and S. Näher. The LEDA Platform of Combinatorial and Geometric Computing. Cambridge University Press, 1999.

[MS96] D.R. Musser and A. Saini. STL Tutorial and Reference Guide. Addison-Wesley, 1996.

[Sit94] M. Sitaraman (coordinator). Special Feature: Component-Based Software using RESOLVE. ACM Software Engineering Notes, 19(4):21-67, October 1994. 


\section{Appendix}

\section{Package specification of Containers}

-- Copyright (C) 1994-1999 Grady Booch, David Weller and Simon Wright.

- All Rights Reserved.

$--$

- - This program is free software; you can redistribute it

- and/or modify it under the terms of the Ada Community

-- License which comes with this Library.

$--$

- $\quad$ This program is distributed in the hope that it will be

-- useful, but WITHOUT ANY WARRANTY; without even the implied

- $\quad$ warranty of MERCHANTABILITY or FITNESS FOR A PARTICULAR

- $\quad$ PURPOSE. See the Ada Community License for more details.

-- You should have received a copy of the Ada Community

-- License with this library, in the file named "Ada Community

-- License" or "ACL". If not, contact the author of this library

- for a copy.

-- \$Id: bc-containers.ads,v 1.7 1999/04/10 14:38:20 simon Exp \$

with Ada.Finalization,

with BC.Smart;

generic

type Item is private;

with function "=" ( $\mathrm{L}, \mathrm{R}$ : Item) return Boolean is <>;

package BC.Containers is

- This package specifies the common protocol of all Container classes.

-- This common protocol consists of Iterators and shortcuts.

type Container is new Ada.Finalization. Controlled with private;

- Active iteration

type shortcut is private;

type Iterator is private;

function "=" (L, R : Shortcut) return Boolean;

function Shortcut To The Last Item Added

(In_The_Container : Container) return shortcut;

function Item_of (The_Shortcut : Shortcut) return Item;

function Defined (The Shortcut: Shortcut) return Boolean;

function New Iterator (For The Container : Container)

return Iterator;

- Return a reset Iterator bound to the specific Container.

procedure Reset (Obj : in out Iterator);

-- Reset the Iterator to the beginning.

procedure Reverse_Reset (Obj : in out Iterator);

- Reset the Iterator to the ending. NEW NEW NEW

procedure Next (Obj : in out Iterator);

- Advance the Iterator to the next Item in the container.

procedure Previous (Obj : in out Iterator);

- NEW NEW NEW

function Is_Done (Obj: Iterator) return Boolean;

-- Return True if there are no more Items in the container.

function Current Item (Obj: Iterator) return Item;

-- Return a copy of the current Item.

generic

with procedure Apply (Elem : in out Item);

In The Iterator : in out Iterator;

procedure Access_Current_Item;

- Call Apply for the Iterator's current Item. 


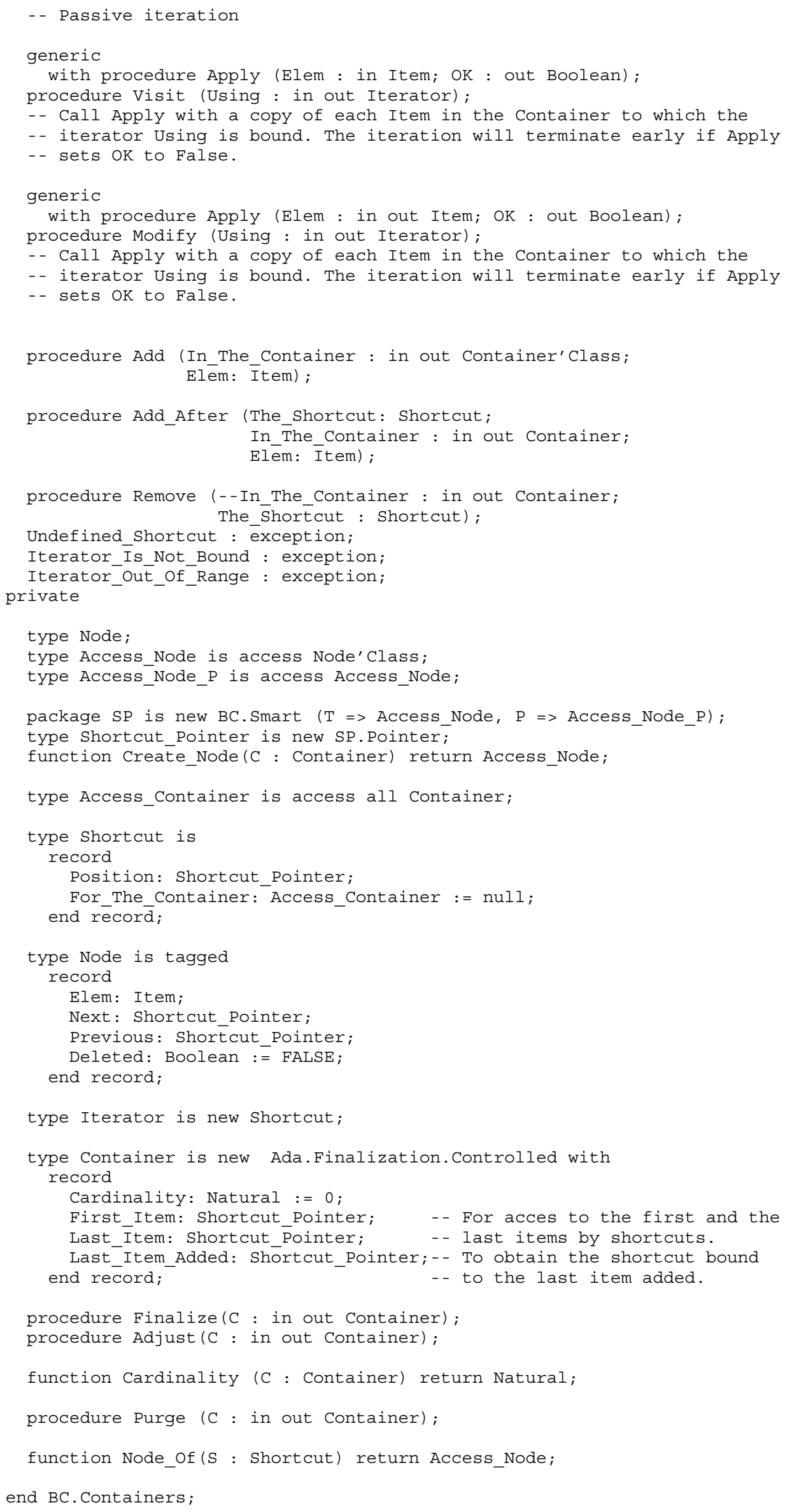




\section{Package body of Containers}

- Copyright (C) 1994-1999 Grady Booch, David Weller and Simon Wright.

- All Rights Reserved.

$-$

- - This program is free software; you can redistribute it

- and/or modify it under the terms of the Ada Community

-- License which comes with this Library.

$--$

- This program is distributed in the hope that it will be

-- useful, but WITHOUT ANY WARRANTY; without even the implied

- warranty of MERCHANTABILITY or FITNESS FOR A PARTICULAR

- PURPOSE. See the Ada Community License for more details.

-- You should have received a copy of the Ada Community

-- License with this library, in the file named "Ada Community

-- License" or "ACL". If not, contact the author of this library

- for a copy.

$--$

-- \$Id: bc-containers.adb,v 1.6 1999/04/10 14:38:20 simon Exp \$

with System.Address_To_Access_Conversions;

package body BC.Containers is

function "=" ( L, R : Shortcut) return Boolean is

begin

return L.Position $=\mathrm{R}$. Position and L.For_The_container $=$ R.For_The_container; end $"="$;

function Create_Node (C : Container) return Access_Node is

begin

return new Node;

end Create_Node;

package Address_Conversions is new System.Address_To_Access_Conversions (Container);

function Shortcut_To_The_Last_Item_Added (In_The_Container : Container) return Shortcut is

$\mathrm{p}$ : Address_Conversions.object Pointer :=

Address_Conversions.To_Pointer (In_The_Container'Address) ;

sh : Shortcut;

begin

sh.Position := In_The_Container.Last_Item_Added;

sh.For_The_Container $\overline{:}=$ Access_Container $(\bar{p})$;

return sh;

end Shortcut_To_The_Last_Item_Added;

procedure Add (In The Container : in out Container'Class; Elem: Item) is

shp, Dummy : Shortcut_Pointer;

begin

shp := Create (new Access_Node' (Create_Node(In_The_Container)));

SP.Value (SP.Pointer (shp)) .all.Elem := Elem;

SP.Value (SP.Pointer (shp)) .all.Previous := In_The_Container.Last_Item;

SP.Value (SP.Pointer (shp)) .all. Next := Dummy;

SP.Value (SP.Pointer (shp)) .all. Deleted := False;

if In_The_Container.Cardinality $=0$ then

else

In_The_Container.First_Item := shp;

SP.Value (SP.Pointer(In_The_Container.Last_Item)).all.Next : = shp; end if;

In_The_Container.Last_Item := shp;

In_The_Container.Last_Item_Added := shp;

In The_Container. Cardinality := In_The_Container. Cardinality + 1 ;

end Ādd; 
procedure Add After (The Shortcut: Shortcut; In The Container : in out Container;

Elem: Item) is

shp : Shortcut_Pointer;

begin

if not Defined(The_Shortcut) then raise Undefined Shortcut;

end if;

if The_Shortcut.Position = In_The_Container.Last_Item then

Add (In The Container, Elem);

else

shp := Create (new Access Node' (Create Node (In The Container))) ;

SP.Value (SP.Pointer (shp)) .all.Elem := Elem;

SP.Value (SP.Pointer (shp)) .all.Previous := The_Shortcut.Position;

SP.Value (SP.Pointer (shp)) .all. Next :=

SP.Value (SP.Pointer(The_Shortcut.Position)).all.Next;

SP.Value (SP.Pointêr (shp)) .all.Deleted := False;

SP.Value (SP.Pointer (SP.Value (SP.Pointer(The_Shortcut.Position)).all.Next)).all.Previous $:=\operatorname{shp} ;$

SP.Value(SP.Pointer(The Shortcut.Position)).all. Next $:=$ shp;

In_The_Container.Last_Item_Added := shp;

In The Container. Cardinality := In The Container. Cardinality + 1;

end if;

end Add_After;

procedure Remove (The_Shortcut : Shortcut) is

Dummy : Shortcut_Pointer;

begin

if not Defined(The_Shortcut) then

raise Undefined Shortcut

end if;

if The_Shortcut.For_The_Container.First_Item = The_Shortcut.Position then

The Shortcut.For The Container.First Item :=

end if : SP.Value (SP. Pointer(The_Short̄cut.Position)).all.Next;

if The_Shortcut.For_The_Container.Last_Item = The_Shortcut.Position then

The_Shortcut.For_The_Container. Last_Item :=

end if; SP.Value(SP.Pointer(The Shortcut.Position)) .all.Previous ;

if The_Shortcut.For_The_Container.Last_Item_Added = The_Shortcut.Position then

The_Shortcut.For_The_Container.Last_Item_Added :=

end if: SP.Value (SP.P̄ointer(The_Shortcut.Position)) .all.Previous;

if SP.Value(SP.Pointer(SP.Value (SP.Pointer(The_shortcut.Position)).all.Next)) /= null then

SP.Value (SP.Pointer(SP.Value (SP.Pointer(The_shortcut.Position)).all.Next)).all.Previous $:=$ end if ;

SP.Value (SP.Pointer(The shortcut.Position)).all.Previous;

if SP.Value(SP.Pointer(SP.Value (SP.Pointer(The shortcut.Position)).all.Previous)) /= null then

SP.Value (SP.Pointer (SP.Value (SP.Pointer(The_shortcut.Position)).all.Previous)).all.Next $:=$ end if;

SP.Value(SP.Pointer(The shortcut.Position)).all.Next

The_Shortcut.For_The_Container.all. Cardinality :=

The Shortcut.For The Container.all.Cardinality - 1

SP.Value (SP.Pointer(The Shortcut.Position)).all.Deleted := True

SP.Value (SP.Pointer(The_Shortcut.Position)) .all. Next := Dummy;

SP.Value(SP.Pointer(The Shortcut.Position)) .all.Previous := Dummy;

end Remove,

function Item of (The Shortcut : Shortcut) return Item is

begin

if not Defined(The Shortcut) then

raise Undefined_s Shortcut;

end if;

return SP.Value(SP.Pointer(The Shortcut.Position)).all.Elem;

end Item of ; 
function Defined (The Shortcut: Shortcut) return Boolean is begin

if SP.Value(SP.Pointer(The Shortcut.Position)) = null then return false;

elsif The_Shortcut.For_The_Container = null then return false:

else return not SP.Value(SP.Pointer(The_Shortcut.Position)).all.Deleted; end if;

end Defined;

function New Iterator (For The Container : Container) return Iterator is

it : Iterator;

$\mathrm{p}$ : Address_Conversions.object_Pointer := begin Address Conversions. To Pointer (For The Container'Address).

it.Position := For The Container.First Item;

it.For The Container := Access Container $(p)$;

return it;

end New_Iterator;

procedure Reset (Obj : in out Iterator) is

begin

if Obj.For_The_Container = null then

raise Itêrator_Is_Not_Bound;

end if;

Obj.Position := Obj.For_The_Container.all.First_Item;

end Reset;

procedure Reverse_Reset (Obj : in out Iterator) is

begin

if Obj.For_The_Container $=$ null then

raise Itêratō_Is_Not_Bound;

end if;

Obj.Position := Obj.For_The_Container.all.Last_Item;

end Reverse Reset;

procedure Next (Obj : in out Iterator) is

begin

if Is_Done (Obj) then

raise Iterator_out_of_Range;

end if;

Obj.Position := SP.Value (SP.Pointer (Obj.Position)).all.Next; end Next;

procedure Previous (Obj : in out Iterator) is

begin

if Is_Done (Obj) then

raise Iterator_out_of_Range;

end if;

Obj.Position := SP.Value (SP.Pointer (Obj.Position)).all.Previous; end Previous;

function Is_Done (Obj : Iterator) return Boolean is

begin

if obj.For_The_Container $=$ null then

raise Itêrator_Is_Not_Bound;

end if;

return SP.Value (SP.Pointer (Obj.Position))= null;

end Is Done;

function Current_Item (Obj : Iterator) return Item is

begin

if Is_Done (Obj) then

raise Iterator out of Range;

end if;

return Item_of $(\mathrm{Obj})$;

end Current_Item;

procedure Access_Current_Item is

begin

Apply (SP.Value (SP.Pointer(In_The_Iterator.Position)).all.Elem); end Access_Current_Item; 


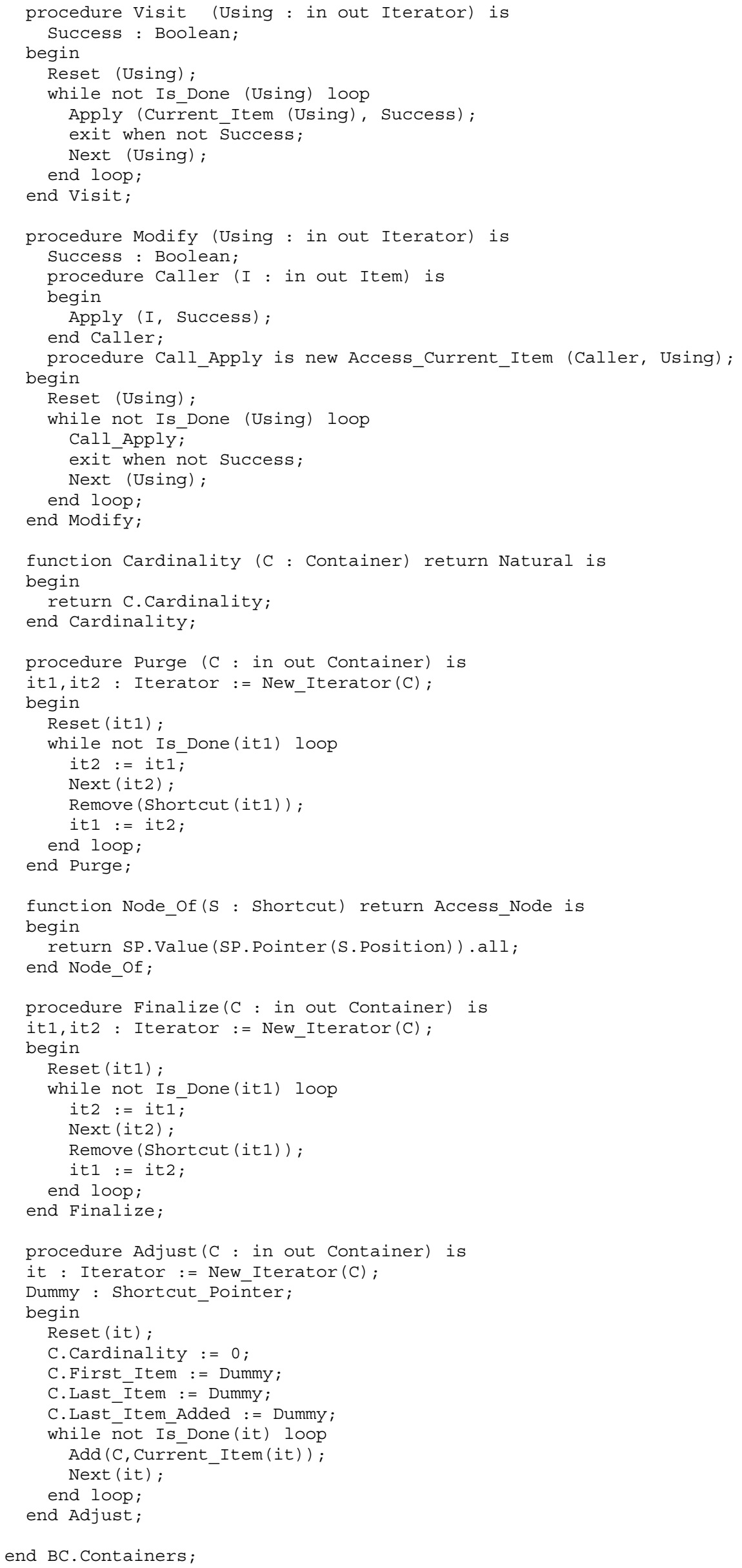




\section{Package specification of Containers.Bags}

- Copyright (C) 1994-1999 Grady Booch and Simon Wright.

- All Rights Reserved.

$--$

- $\quad$ This program is free software; you can redistribute it

- and/or modify it under the terms of the Ada Community

-- License which comes with this Library.

$--$

-- This program is distributed in the hope that it will be

-- useful, but WITHOUT ANY WARRANTY; without even the implied

- warranty of MERCHANTABILITY or FITNESS FOR A PARTICULAR

- $\quad$ PURPOSE. See the Ada Community License for more details.

- - You should have received a copy of the Ada Community

- License with this library, in the file named "Ada Community

-- License" or "ACL". If not, contact the author of this library

- for a copy.

$-$

-- \$Id: bc-containers-bags.ads,V 1.2 1999/04/10 14:38:18 simon Exp \$

generic

package BC.Containers.Bags is

- A bag denotes a collection of items, drawn from some well-defined

-- universe. A bag may contain duplicate items. A bag actually owns only

-- one copy of each unique item: duplicates are counted, but are not

- stored with the bag.

- The parameter Item denotes the universe from which the bag draws its

- items. Items may be a primitive type or user-defined.

type Bag is abstract new Container with private;

function Are_Equal ( $L, R$ : Bag'Class) return Boolean;

-- Return True if and only if both bags have the same number of distinct

- items, and the same items themselves, each with the same count; return

- False otherwise.

- Can't call this "=" because of the standard one for Bag.

procedure Clear (B : in out Bag) is abstract;

- Empty the bag of all items.

procedure Add (B : in out Bag; I : Item; Added : out Boolean) is abstract; - Add the item to the bag. If the item is not already a distinct member

- of the bag, copy the item and add it to the bag and set Added to

- True. If the item already exists, then increment the number of that

- item and set Added to False.

procedure Add (B : in out Bag'Class; I : Item);

- Add the item to the bag. If the item is not already a distinct member

-- of the bag, copy the item and add it to the bag; if it is, increment

-- the number of that item.

procedure Remove (B : in out Bag; I : Item) is abstract;

-- If the item is not a member of the bag, raise BC.Not Found. Otherwise,

- - if there is exactly one of the item in the bag, remove the item in the

-- bag; if there is more than one of the item in the bag, simply decrement

- its number.

procedure Union (B : in out Bag'Class; O : Bag'Class);

- Perform a logical bag union; at the completion of this operation, the

- bag B contains the items and counts found in its original state

- combined with the bag $O$. For each item in the bag o, if the item is

- not already a distinct member of the bag $S$, copy the item and add it

- - and its count to the bag $S$. If the item already is a member, increment

- its count in $S$.

procedure Intersection (B : in out Bag'Class; O : Bag'Class);

-- Perform a logical bag intersection; at the completion of this

- operation, the bag B contains the items found both in its original

- state and in the bag $O$. For each item in the bag 0 , if the item is not

- already a distinct member of the bag $S$, do nothing. If the item

- already is a member of $S$, set its count to the lower of the two

- counts. Items in the bag $S$ but not in the bag 0 are also removed. 
procedure Difference (B : in out Bag'Class; O : Bag'Class);

-- Perform a logical bag difference; at the completion of this operation,

-- the bag s contains the items found in its original state, less those

- found in the bag $O$. For each item in the bag 0 , if the item is not

- already a distinct member of the bag $S$, do nothing. If the item is a

-- member of the bag $S$ with a count less than that in the bag O, remove

- - the item from the bag $S$. If the item is a member of the bag $S$ with a

-- count more than that in the bag $O$, decrement the count in the bag $S$ by

- the count in the bag 0 .

function Extent (B : Bag) return Natural is abstract;

-- Return the number of distinct items in the bag.

function Total_Size (B : Bag'Class) return Natural;

-- Return the total number of items in the bag.

function Count (B : Bag; I : Item) return Natural is abstract;

-- Return the number of times the item occurs in the bag.

function Is_Empty (B : Bag) return Boolean is abstract;

-- Return True if and only if there are no items in the bag.

function Is_Member (B : Bag; I : Item) return Boolean is abstract;

-- Return True if and only if the item exists in the bag.

function Is_Subset (B : Bag'Class; O : Bag'Class) return Boolean;

-- Return True if and only if the bag B has the same or fewer distinct

- items than in the bag $O$ and equal or less numbers of each such item

- than in the bag 0 .

function Is_Proper_Subset (B : Bag'Class; O : Bag'Class) return Boolean;

- Return True if and only if

- - all the distinct items in the bag $B$ are also in the bag 0 , and

-- either at least one of the items in the bag B has a lower number

- than the number in the bag 0 ,

- or there is at least one distinct item in the bag o that is not

- in the bag $B$.

private

type Bag is abstract new Container with null record;

procedure Attach (B : in out Bag; I : Item; C : Positive);

procedure Detach (B : in out Bag; I : Item);

procedure Set_Value (B : in out Bag; I : Item; C : Positive);

function Multiplicity (B : Bag'Class) return Natural;

function Number_of_Buckets (B : Bag) return Natural;

function Length ( ${ }^{-}$: Bag; Bucket : Positive) return Natural;

function Exists (B : Bag; I : Item) return Boolean;

function Item_At (B : Bag; Bucket, Index : Positive) return Item_Ptr;

function Value At (B : Bag; Bucket, Index : Positive) return Positive;

type Bag_Iterator (B : access Bag'Class)

is new Actual Iterator (B) with record

Bucket_Index : Natural :=0;

Index : Natural :=0 ;

end record;

procedure Initialize (It : in out Bag_Iterator);

-- Overriding primitive supbrograms of the concrete actual Iterator.

procedure Reset (It : in out Bag_Iterator);

procedure Next (It : in out Bag_Iterator);

function Is Done (It : Bag Iterator) return Boolean;

function Current_Item (It : Bag_Iterator) return Item;

function Current_Item (It : Bag_Iterator) return Item_Ptr;

end BC.Containers.Bags; 


\section{Package body of Containers.Bags}

-- Copyright (C) 1994-1999 Grady Booch and Simon Wright.

- All Rights Reserved.

$--$

- - This program is free software; you can redistribute it

-- and/or modify it under the terms of the Ada Community

-- License which comes with this Library.

$--$

-- This program is distributed in the hope that it will be

-- useful, but WITHOUT ANY WARRANTY; without even the implied

-- warranty of MERCHANTABILITY or FITNESS FOR A PARTICULAR

- $\quad$ PURPOSE. See the Ada Community License for more details.

-- You should have received a copy of the Ada Community

-- License with this library, in the file named "Ada Community

-- License" or "ACL". If not, contact the author of this library

- for a copy.

$--$

-- \$Id: bc-containers-bags.adb,v 1.2 1999/04/10 14:38:18 simon Exp \$

with BC.Support. Exceptions;

with System.Address_To_Access_Conversions;

package body BC.Containers.Bags is

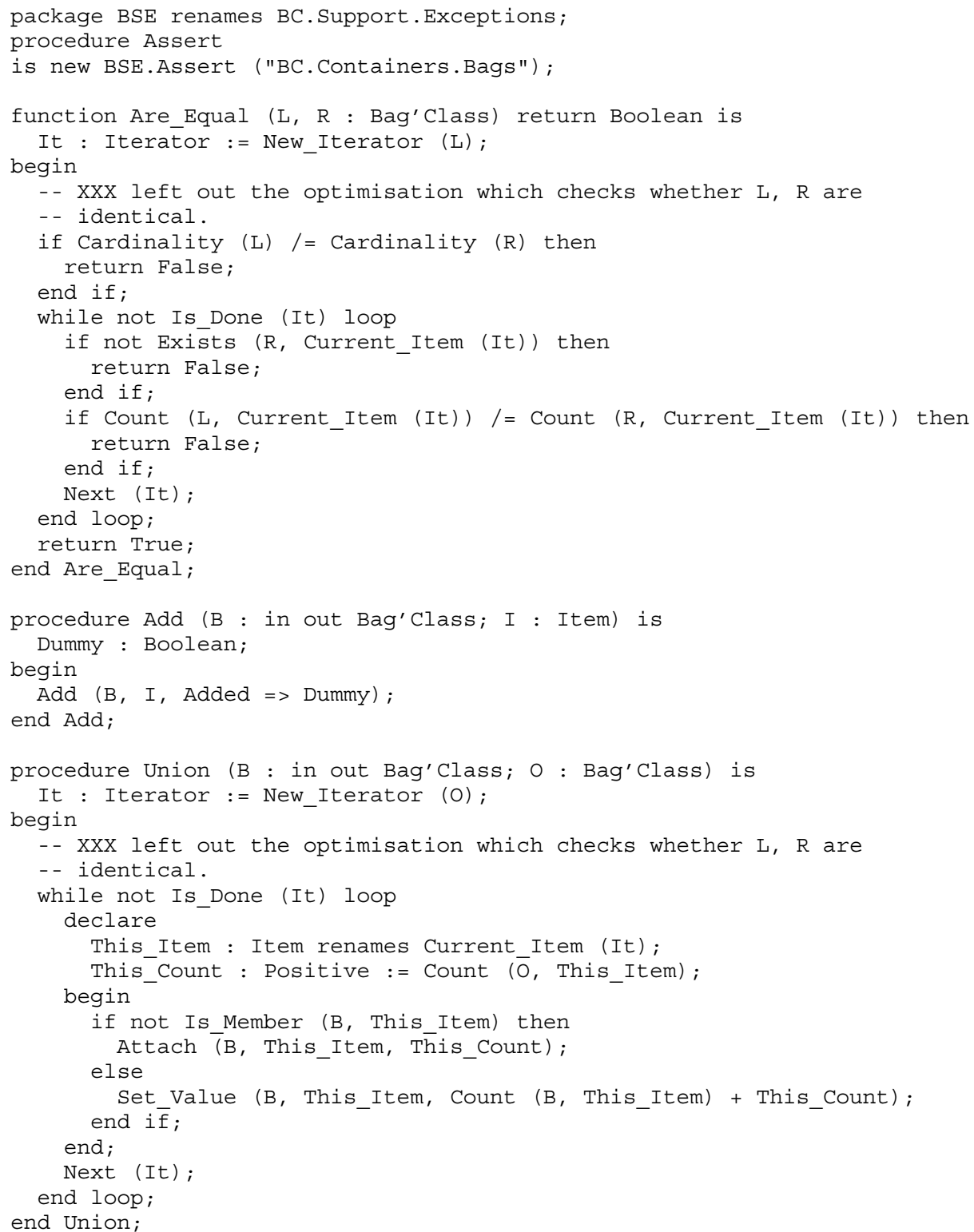




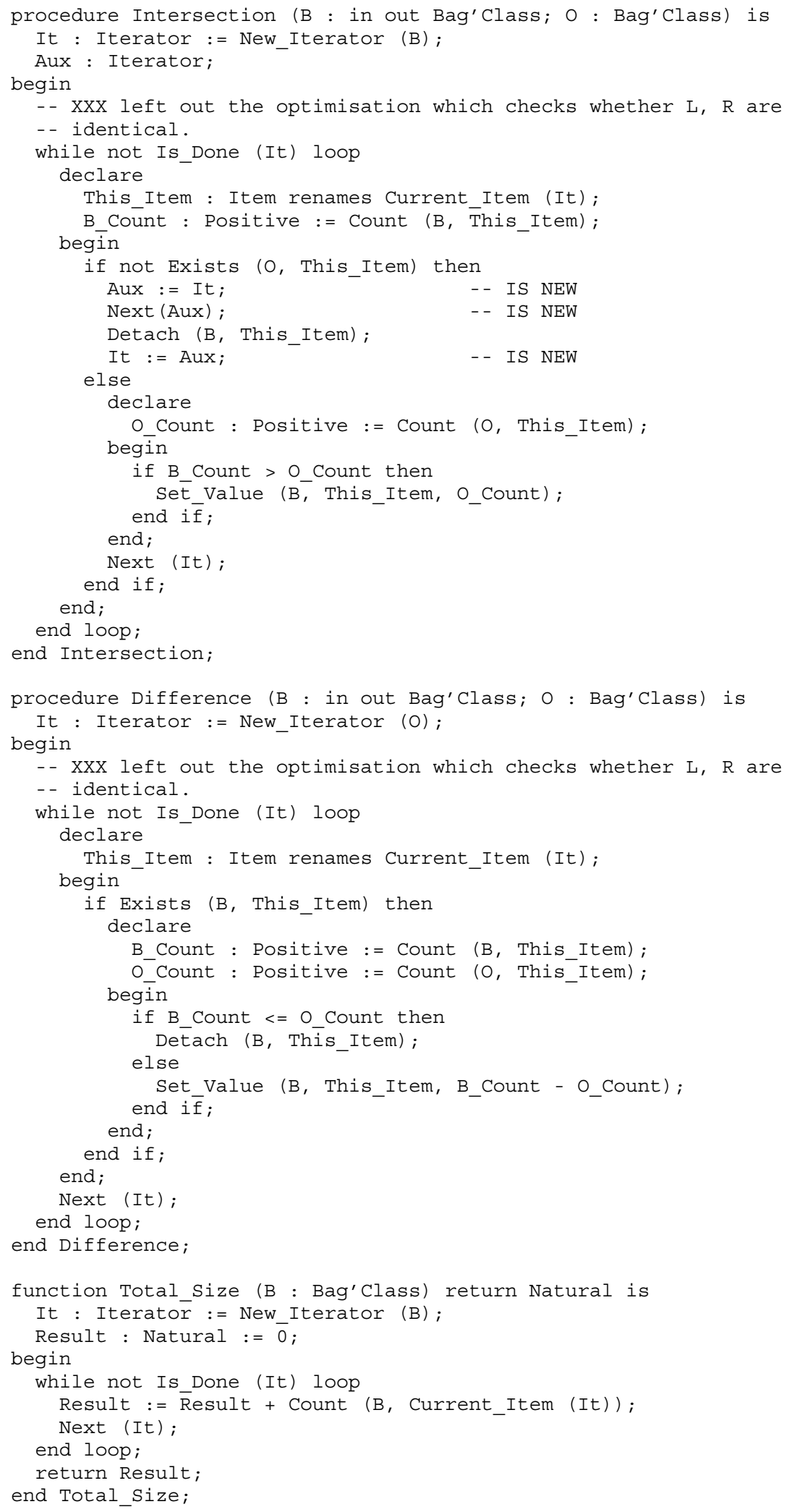




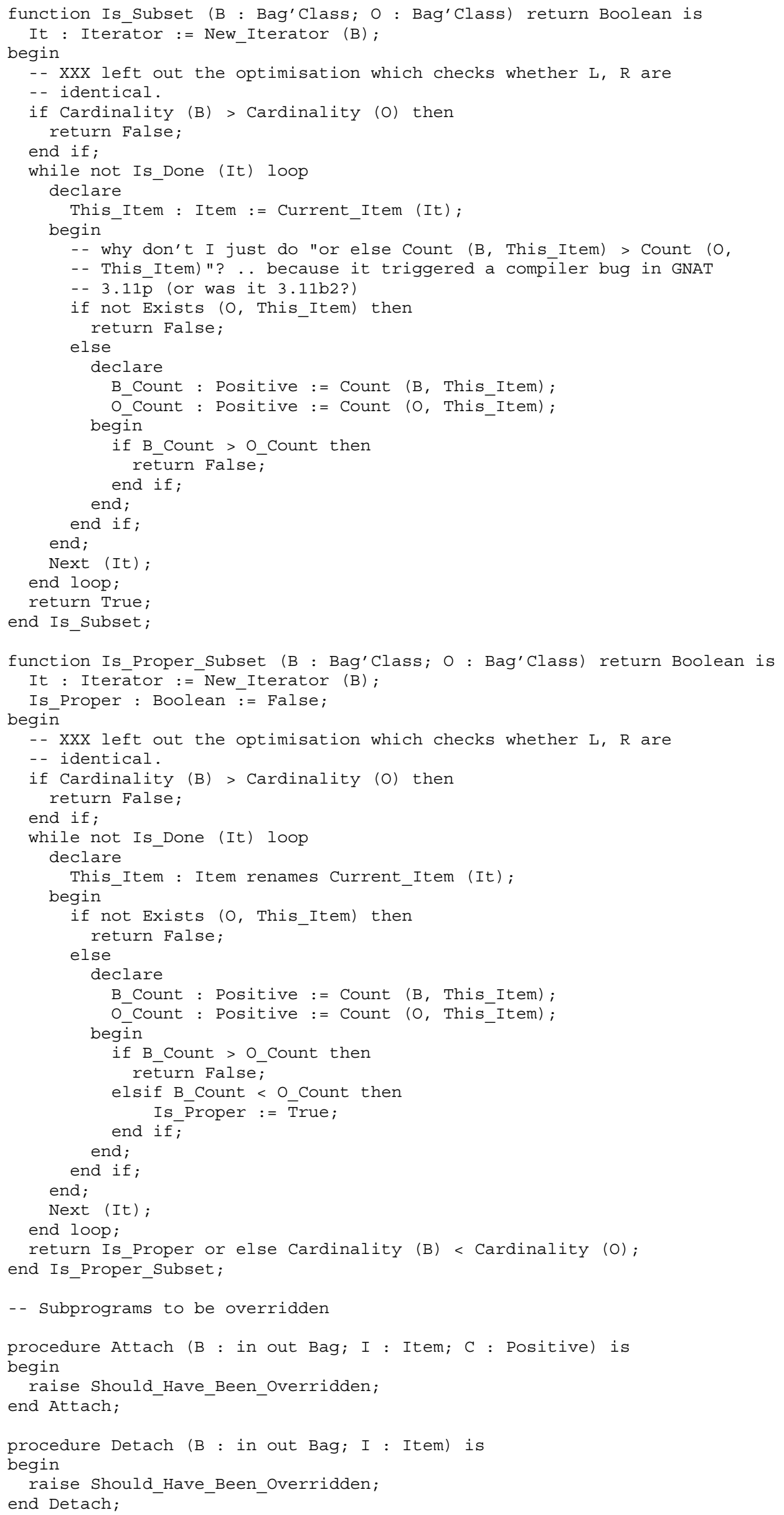




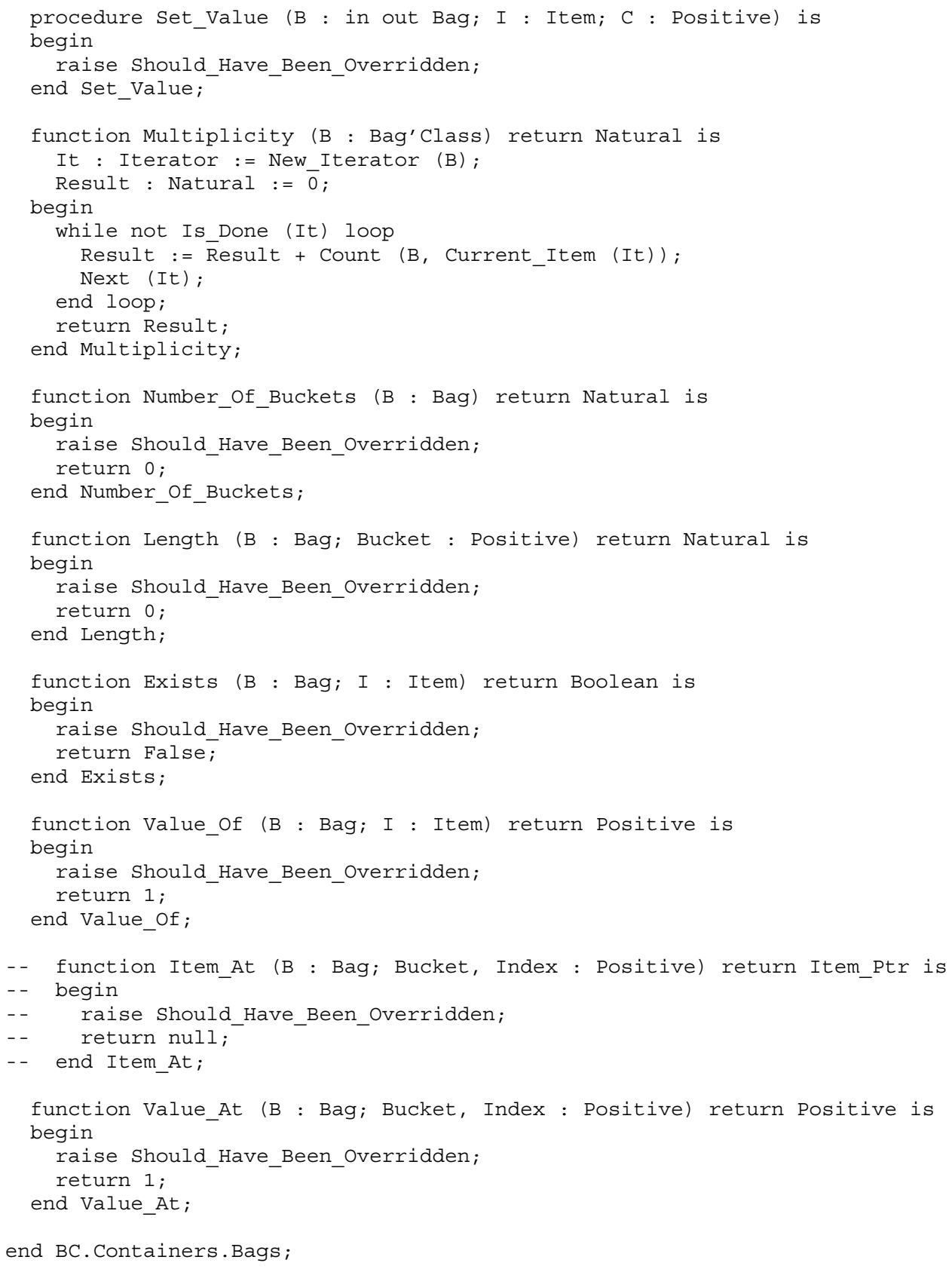




\section{Package specification of Containers.Bags.Bounded}

- Copyright (C) 1994-1999 Grady Booch and Simon Wright.

- All Rights Reserved.

-

- - This program is free software; you can redistribute it

- and/or modify it under the terms of the Ada Community

-- License which comes with this Library.

$--$

-- This program is distributed in the hope that it will be

-- useful, but WITHOUT ANY WARRANTY; without even the implied

- $\quad$ warranty of MERCHANTABILITY or FITNESS FOR A PARTICULAR

- $\quad$ PURPose. See the Ada Community License for more details.

-- You should have received a copy of the Ada Community

-- License with this library, in the file named "Ada Community

-- License" or "ACL". If not, contact the author of this library

- for a copy.

-- \$Id: bc-containers-bags-bounded.ads,V 1.2 1999/04/10 14:38:18 simon Exp \$

with BC.Support. Bounded;

with BC.Support.Hash_Tables;

generic

with function Hash (V : Item) return Positive is <>;

Buckets : Positive;

Size : Positive;

package BC.Containers.Bags.Bounded is

- A bag denotes a collection of items, drawn from some well-defined

- - universe. A bag may contain duplicate items. A bag actually owns only

-- one copy of each unique item: duplicates are counted, but are not

- stored with the bag.

- - The hash function (the generic parameter Hash) determines the

- allocation of pairs to hash buckets. The value returned must not

- change during the lifetime of a given Item. The range of hash values

-- need not be constrained to the number of buckets in the bag.

-- The hash function must satisfy the condition that, for objects $A$ and

-- $B$, if $A=B$, then Hash (A) must equal Hash (B). The hash function

- should attempt to spread the set of possible items uniformly across

- the number of buckets. The quality of the hash function has a

- significant impact upon performance.

type Bounded_Bag is new Bag with private;

procedure Clear (B : in out Bounded_Bag);

- Empty the bag of all items.

procedure Add (B : in out Bounded Bag; I : Item; Added : out Boolean);

- Add the item to the bag. If the item is not already a distinct member

-- of the bag, copy the item and add it to the bag and set Added to

-- True. If the item already exists, then increment the number of that

- item and set Added to False.

function Available (B : Bounded_Bag) return Natural;

- Return the number of unused slots in the bag. Note, since hash buckets

- - are of fixed size in the bounded bag it will probably not be possible

- to use all these slots.

procedure Remove (B : in out Bounded_Bag; I : Item);

- - If the item is not a member of the bag, raise BC.Not_Found. Otherwise,

-- if there is exactly one of the item in the bag, remove the item in the

- bag; if there is more than one of the item in the bag, simply decrement

- its number.

function Extent (B : Bounded_Bag) return Natural;

-- Return the number of distinct items in the bag.

function Count (B : Bounded Bag; I : Item) return Natural;

-- Return the number of times the item occurs in the bag.

function Is_Empty (B : Bounded_Bag) return Boolean;

-- Return True if and only if there are no items in the bag. 
function Is_Member (B : Bounded_Bag; I : Item) return Boolean; -- Return True if and only if the item exists in the bag.

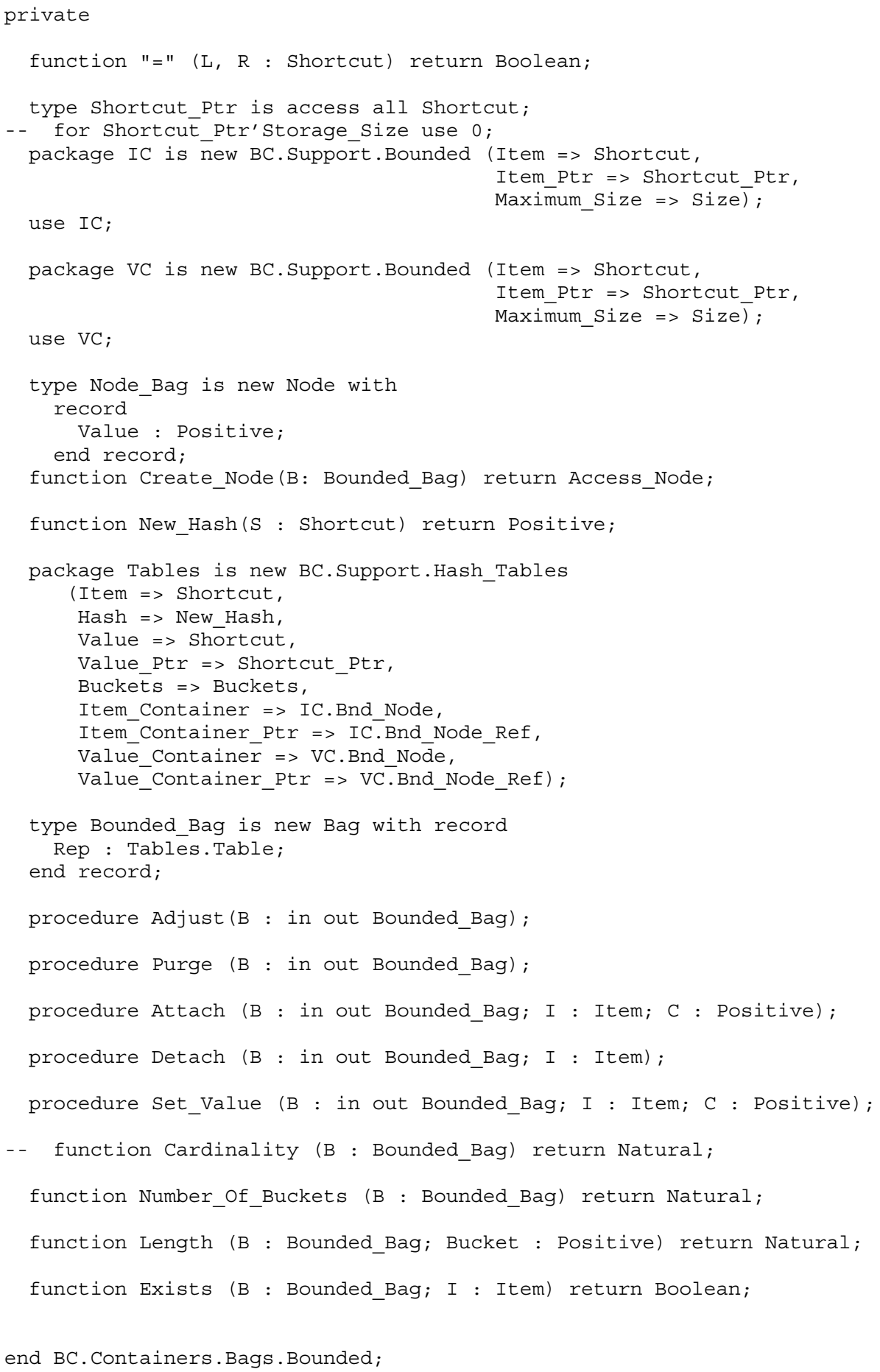




\section{Package body of Containers.Bags.Bounded}

- Copyright (C) 1994-1999 Grady Booch and Simon Wright.

- All Rights Reserved.

$--$

- $\quad$ This program is free software; you can redistribute it

- $\quad$ and/or modify it under the terms of the Ada Community

-- License which comes with this Library.

$--$

- This program is distributed in the hope that it will be

-- useful, but WITHOUT ANY WARRANTY; without even the implied

- $\quad$ warranty of MERCHANTABILITY or FITNESS FOR A PARTICULAR

- $\quad$ PURPose. See the Ada Community License for more details.

- $\quad$ You should have received a copy of the Ada Community

- License with this library, in the file named "Ada Community

-- License" or "ACL". If not, contact the author of this library

- $\quad$ for a copy.

-- \$Id: bc-containers-bags-bounded.adb,v 1.2 1999/04/10 14:38:18 simon Exp \$

with BC.Support. Exceptions;

with System.Address_To_Access_Conversions;

--with Ada.Text_Io; usēe Ada.Text_ı;

package body BC.Containers.Bags. Bounded is

package BSE renames BC.Support.Exceptions;

procedure Assert

is new BSE.Assert ("BC.Containers.Bags.Bounded");

function Create_Node(B : Bounded_Bag) return AcCess_Node is

A : Access Node;

begin

A : = new Node Bag;

Node Bag $($ A.all $)$. Value $:=1$,

return A;

end Create Node;

function "=" ( $\mathrm{L}, \mathrm{R}$ : Shortcut) return Boolean is

begin

return Item_of $(\mathrm{L})=$ Item_of $(\mathrm{R})$;

end $"="$;

function New_Hash (S : Shortcut) return Positive is

begin

return Hash (Item_of (S));

end New_Hash;

procedure Clear ( $B$ : in out Bounded_Bag) is

begin

Purge (Container (B));

Tables.Clear (B.Rep);

end Clear;

procedure Add (B : in out Bounded_Bag; I : Item; Added : out Boolean) is

sh : Shortcut;

A : Access_Node;

begin

Containers. Add (B, I) ;

sh := Shortcut_To_The_Last_Item_Added (B);

if Tables.Is Bound (B. $\bar{R} e p, s \bar{h}$ ) then

sh := Tablēs.Value_of (B.Rep, sh);

A : = Node_of (sh);

Node Bag (A.all). Value $:=\operatorname{Node~Bag(A.all).Value~+~} 1$

Adde $\bar{d}:=$ False;

Remove (Shortcut To The Last Item Added(B));

B. Last_Item_Added $\overline{:}=\mathrm{sh}$.Position;

else

Tables.Bind (B.Rep, sh, sh);

Added := True;

end if:

end Add; 


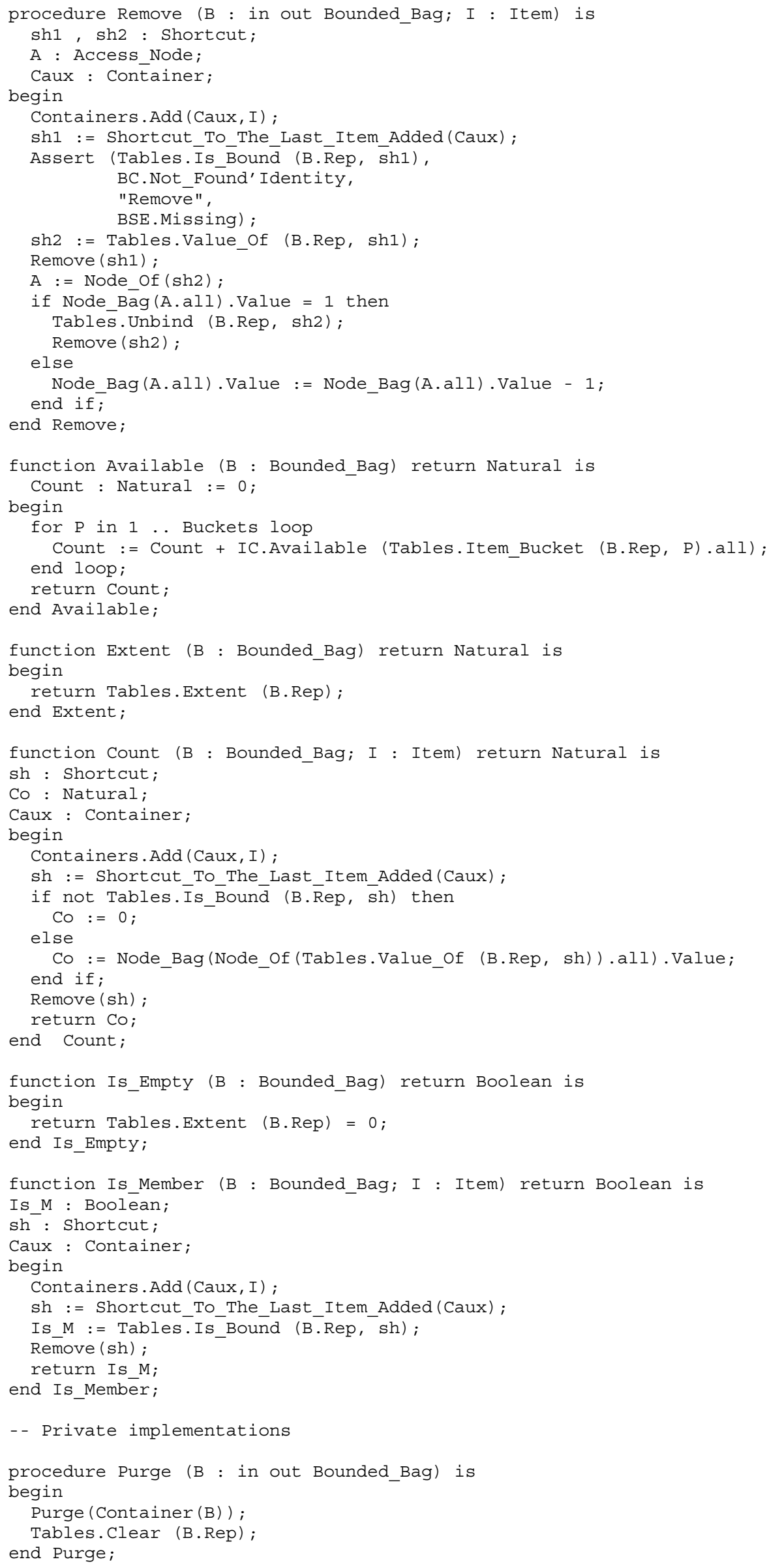




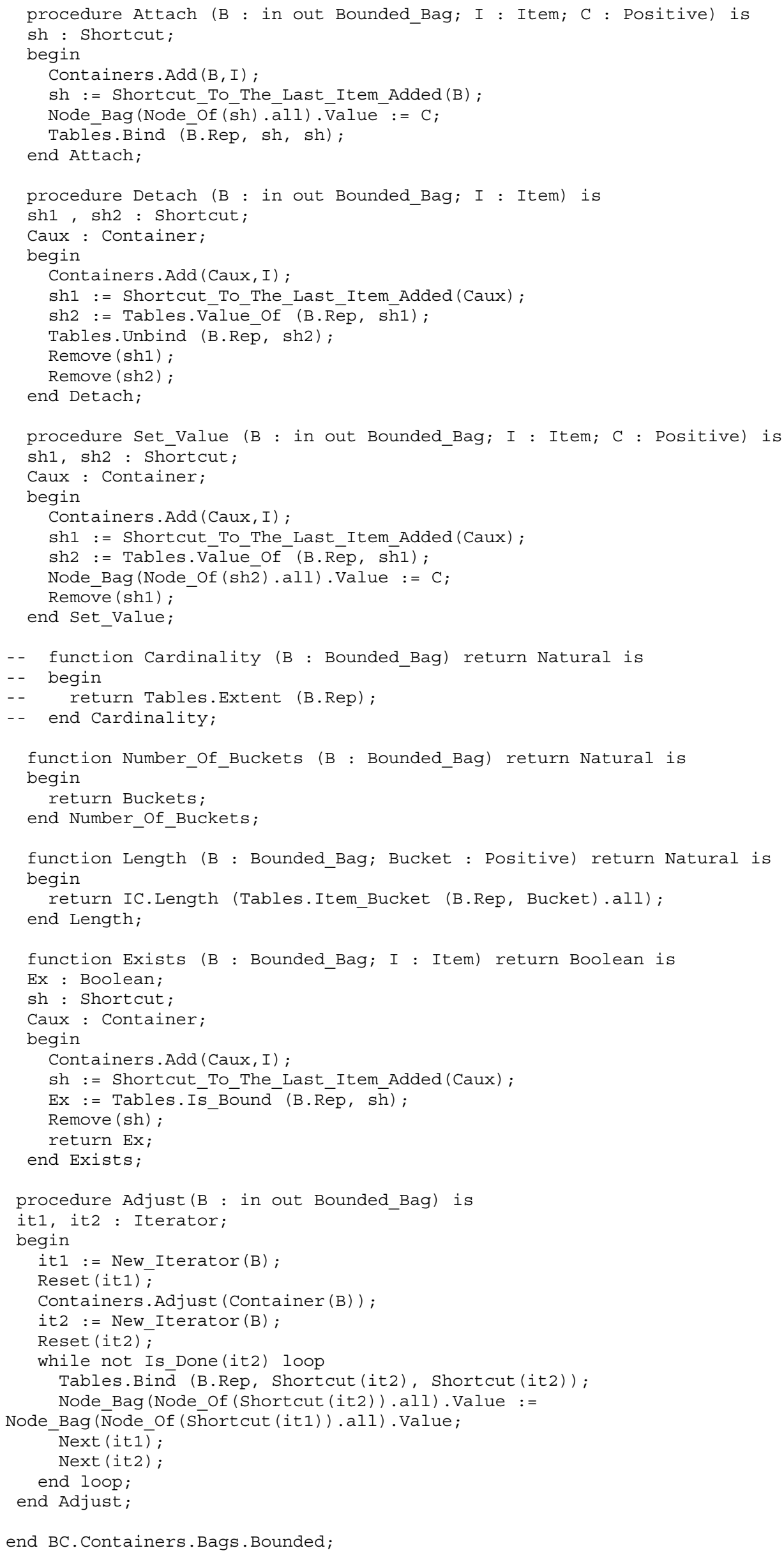

